\title{
Asymptotic Dirichlet problems in warped products
}

\author{
Jean-Baptiste Casteras ${ }^{1}$ - Esko Heinonen ${ }^{2} \cdot$ Ilkka Holopainen $^{2} \cdot$ Jorge Lira $^{3}$
}

Received: 6 April 2018 / Accepted: 12 May 2019 / Published online: 26 June 2019

(c) The Author(s) 2019

\begin{abstract}
We study the asymptotic Dirichlet problem for Killing graphs with prescribed mean curvature $H$ in warped product manifolds $M \times_{\varrho} \mathbb{R}$. In the first part of the paper, we prove the existence of Killing graphs with prescribed boundary on geodesic balls under suitable assumptions on $H$ and the mean curvature of the Killing cylinders over geodesic spheres. In the process we obtain a uniform interior gradient estimate improving previous results by Dajczer and de Lira. In the second part we solve the asymptotic Dirichlet problem in a large class of manifolds whose sectional curvatures are allowed to go to 0 or to $-\infty$ provided that $H$ satisfies certain bounds with respect to the sectional curvatures of $M$ and the norm of the Killing vector field. Finally we obtain non-existence results if the prescribed mean curvature function $H$ grows too fast.
\end{abstract}

Keywords Mean curvature equation · Killing graph · Dirichlet problem · Hadamard manifold · warped product

Mathematics Subject Classification Primary 58J32; Secondary 53C21

Jean-Baptiste Casteras supported by MIS F.4508.14 (FNRS). Esko Heinonen supported by Jenny and Antti Wihuri Foundation and CNPq. Ilkka Holopainen supported by the Faculty of Science, University of Helsinki, and FUNCAP. Jorge Lira supported by CNPq and FUNCAP.

$凶 \quad$ Ilkka Holopainen

ilkka.holopainen@helsinki.fi

Jean-Baptiste Casteras

jeanbaptiste.casteras@gmail.com

Esko Heinonen

esko.heinonen@helsinki.fi

Jorge Lira

jorge.lira@mat.ufc.br

1 Departement de Mathematique, Universite libre de Bruxelles, CP 214, Boulevard du Triomphe, 1050 Bruxelles, Belgium

2 Department of Mathematics and Statistics, University of Helsinki, P.O.B. 68, Pietari Kalmin katu 5, 00014 Helsinki, Finland

3 Departamento de Matematica, Universidade Federal do Ceará, Bloco 914, Campus do Pici, Fortaleza, Ceará 60455-760, Brazil 


\section{Contents}

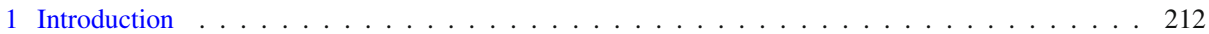

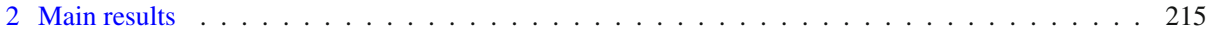

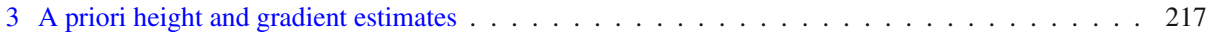

3.1 Height estimate . . . . . . . . . . . . . . . . . . . . . . . . . 218

3.2 Boundary gradient estimate . . . . . . . . . . . . . . . . . . . . . . . . . . . 219

3.3 Interior gradient estimate . . . . . . . . . . . . . . . . . . . . . . . . 223

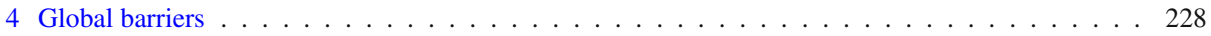

4.1 Example: hyperbolic space . . . . . . . . . . . . . . . . . . . . . . 233

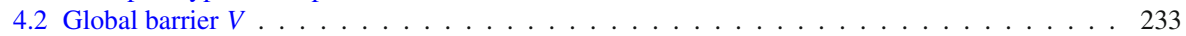

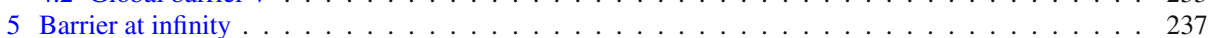

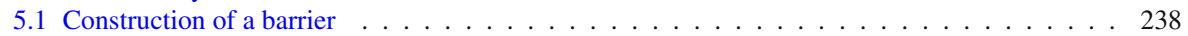

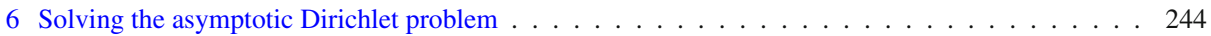

7 Non-existence result . . . . . . . . . . . . . . . . . . . . . . . . . . . 246

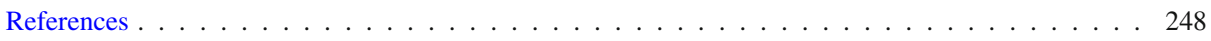

\section{Introduction}

Let $N$ be a Riemannian manifold of the form $N=M \times{ }_{\varrho} \mathbb{R}$, where $M$ is a complete $n$ dimensional Riemannian manifold and $\varrho \in C^{\infty}(M)$ is a smooth (warping) function. This means that the Riemannian metric $\bar{g}$ in $N$ is of the form

$$
\bar{g}=\left(\varrho \circ \pi_{1}\right)^{2} \pi_{2}^{*} \mathrm{~d} t^{2}+\pi_{1}^{*} g,
$$

where $g$ denotes the Riemannian metric in $M$ whereas $t$ is the natural coordinate in $\mathbb{R}$ and $\pi_{1}: M \times \mathbb{R} \rightarrow M$ and $\pi_{2}: M \times \mathbb{R} \rightarrow \mathbb{R}$ are the standard projections. It follows that the coordinate vector field $X=\partial_{t}$ is a Killing field and that $\varrho=|X|$ on $M$. Since the norm of $X$ is preserved along its flow lines, we may extend $\varrho$ to a smooth function $\varrho=|X| \in C^{\infty}(N)$. From now on, we suppose that $\varrho>0$ on $M$.

In this paper we study Killing graphs with prescribed mean curvature. Such graphs were introduced by Dajczer and Ripoll in [9], where the Dirichlet problem for a graph of constant mean curvature $H$ with $C^{2, \alpha}$ boundary values was solved in a bounded domain $\Omega$ contained in a normal geodesic disk $D \subset M$ of radius $r_{0}$ under hypothesis involving $r_{0}$, data on $\Omega$, and the curvature of the ambient 3-dimensional space $N$. A bit later in [10] the Dirichlet problem for prescribed mean curvature $H \in C^{\alpha}$ with $C^{2, \alpha}$ boundary values was solved in bounded domains $\Omega \subset M$ with $C^{2, \alpha}$ boundary again under hypothesis involving data on $\Omega$ and the Ricci curvature of the ambient space $N$. Recall that given a domain $\Omega \subset M$, the Killing graph of a $C^{2}$ function $u: \Omega \rightarrow \mathbb{R}$ is the hypersurface given by

$$
\Sigma_{u}=\{(x, u(x)): x \in \Omega\} \subset M \times \mathbb{R} .
$$

In other words,

$$
\Sigma_{u}=\{\Psi(x, u(x)): x \in \Omega\},
$$

where $\Psi: \Omega \times \mathbb{R} \rightarrow N$ is the flow generated by $X$. In [11] the Dirichlet problem was solved with merely continuous boundary data. Furthermore, the authors proved the existence and uniqueness of so-called radial graphs in the hyperbolic space $\mathbb{H}^{n+1}$ with prescribed mean curvature and asymptotic boundary data at infinity thus solving the asymptotic Dirichlet problem in $\mathbb{H}^{n} \times \cosh r \mathbb{R}$. One of our goals in the current paper is to solve the asymptotic Dirichlet problem with prescribed mean curvature in a large class of negatively curved manifolds. 
On the other hand, it is an interesting question under which conditions on a Riemannian manifold $M$ every entire constant mean curvature graph over $M$ is a slice, i.e. a graph of a constant function. The first such result is the celebrated theorem due to Bombieri, De Giorgi, and Miranda [3] that an entire minimal positive graph over $\mathbb{R}^{n}$ is a totally geodesic slice. Their result was extended by Rosenberg, Schulze, and Spruck [18] to a complete Riemannian manifold $M$ with nonnegative Ricci curvature and the sectional curvature bounded from below by a negative constant. Ding, Jost, and Xin considered in [12] complete, noncompact Riemannian manifolds with nonnegative Ricci curvature, Euclidean volume growth, and quadratic decay of the curvature tensor. They proved that an entire minimal graph over such a manifold $M$ must be a slice if its height function has at most linear growth on one side unless $M$ is isometric to Euclidean space. In the recent paper [5] Casteras, Heinonen, and Holopainen showed that a minimal positive graph over a complete Riemannian manifold with asymptotically nonnegative sectional curvature and only one end is a slice if its height function has at most linear growth. Entire Killing graphs in $M \times_{\varrho} \mathbb{R}$ with constant mean curvature were studied in [7,8]. In particular, it was shown in [7] that a bounded entire Killing graph of constant mean curvature must be a slice if $\operatorname{Ric}_{M} \geq 0, K_{M} \geq-K_{0}$ for some $K_{0} \geq 0$, and if $\varrho \geq \varrho_{0}>0$, with $\|\varrho\|_{C^{2}(M)}<\infty$.

Our current paper is inspired by the above mentioned research [7,8,10,11] on Killing graphs with prescribed mean curvature as well as by the recent paper [4]. In the latter, the asymptotic Dirichlet problem for $f$-minimal graphs in Cartan-Hadamard manifolds $M$ has been studied. Recall that $f$-minimal hypersurfaces are natural generalizations of selfshrinkers which play a crucial role in the study of mean curvature flow. Moreover, they are minimal hypersurfaces of weighted manifolds $M_{f}=\left(M, g, e^{-f} \mathrm{~d} \mathrm{vol}_{M}\right)$, where $(M, g)$ is a complete Riemannian manifold with the Riemannian volume element $\mathrm{d} \operatorname{vol}_{M}$.

Returning to the Killing graph $\Sigma_{u}$ of a function $u$, we note that the induced metric in $\Sigma_{u}$ has components

$$
g_{i j}+\varrho^{2}(x) u_{i} u_{j},
$$

where $g_{i j}$ are local components of the metric $g$. The induced volume element in $\Sigma_{u}$ (or equivalently, on the domain $\Omega \subset M$ ) is given by

$$
\mathrm{d} \Sigma=\varrho \sqrt{\varrho^{-2}+|\nabla u|^{2}} \mathrm{~d} M .
$$

We consider the constrained area functional

$$
\mathcal{A}_{H}[u]=\int_{\Omega} \varrho \sqrt{\varrho^{-2}+|\nabla u|^{2}} \mathrm{~d} M+\nu_{H}[u],
$$

where

$$
\nu_{H}[u]=\int_{\Omega} \int_{0}^{u(x) \varrho(x)} n H \mathrm{~d} M=\int_{\Omega} n H \varrho u \mathrm{~d} M
$$

and $H$ is a smooth function on $\Omega$. Given an arbitrary compactly supported function $v \in$ $C_{0}^{\infty}(\Omega)$ we have the first variation formula

$$
\delta \mathcal{A}_{H}[u] \cdot v=\left.\frac{\mathrm{d}}{\mathrm{d} s}\right|_{s=0} \mathcal{A}_{H}[u+s v]=-\int_{\Omega}\left(\operatorname{div}\left(\frac{\nabla u}{W}\right)+\left\langle\nabla \log \varrho, \frac{\nabla u}{W}\right\rangle-n H\right) v \varrho \mathrm{d} M,
$$

where

$$
W=\sqrt{\varrho^{-2}+|\nabla u|^{2}}
$$


and the differential operators $\nabla$ and div are taken with respect to the metric $g$ in $M$. Then the Euler-Lagrange equation of this functional is

$$
\operatorname{div}\left(\frac{\nabla u}{W}\right)+\left\langle\nabla \log \varrho, \frac{\nabla u}{W}\right\rangle=n H
$$

and $H(x)$ is the mean curvature of the graph $\Sigma_{u} \subset M \times_{\varrho} \mathbb{R}$ at $(x, u(x))$. The equation (1.3) can be rewritten as

$$
\operatorname{div}_{-\log \varrho}\left(\frac{\nabla u}{W}\right)=n H,
$$

where the weighted divergence operator corresponding to a smooth density function $f \in$ $C^{\infty}(M)$ is defined by

$$
\operatorname{div}_{f} Z=e^{f} \operatorname{div}\left(e^{-f} Z\right)=\operatorname{div} Z-\langle\nabla f, Z\rangle .
$$

Note that this is the divergence-form operator that fits well with the weighted measure $\varrho \mathrm{d} M$ in the sense that a suitable version of the divergence theorem is still valid in this context. Reasoning another way around, since $\Sigma$ is oriented by the normal vector field

$$
N=\frac{1}{W}\left(\varrho^{-2} X-\left.\nabla u\right|_{(x, u(x))}\right)
$$

and

$$
\left\langle\nabla \log \varrho, \frac{\nabla u}{W}\right\rangle=-\langle\bar{\nabla} \log \varrho, N\rangle
$$

where $\bar{\nabla}$ is the Riemannian connection in $N$, we can interpret

$$
H_{\log \varrho}=H+\frac{1}{n}\langle\nabla \log \varrho, N\rangle
$$

as a weighted mean curvature of the submanifold $\Sigma_{u}$ in the Riemannian product $M \times \mathbb{R}$ in the sense that the Euler-Lagrange PDE may be rewritten as

$$
\operatorname{div}\left(\frac{\nabla u}{W}\right)=n H_{\log \varrho} .
$$

More generally, if $f$ is an arbitrary density in $M$ we consider a weighted area functional of the form

$$
\mathcal{A}_{H, f}[u]=\int_{\Omega} e^{-f} \varrho \sqrt{\varrho^{-2}+|\nabla u|^{2}} \mathrm{~d} M+\int_{\Omega} n H e^{-f} \varrho u \mathrm{~d} M .
$$

In this case, the Euler-Lagrange equation is

$$
\operatorname{div}_{f}\left(\frac{\nabla u}{W}\right)+\left\langle\nabla \log \varrho, \frac{\nabla u}{W}\right\rangle=n H .
$$

As before, this equation may be rewritten either in terms of a modified weighted divergence

$$
\operatorname{div}_{f-\log \varrho}\left(\frac{\nabla u}{W}\right)=n H
$$

or as a prescribed weighted mean curvature equation

$$
\operatorname{div}_{f}\left(\frac{\nabla u}{W}\right):=\operatorname{div}\left(\frac{\nabla u}{W}\right)+\langle\bar{\nabla} f, N\rangle=n H_{\log \varrho} .
$$


For the time being, we restrict ourselves to the case where $f=0$. Intrinsically, given a hypersurface $\Sigma \subset N$ and denoting $u=\left.t\right|_{\Sigma}$, the parametric counterpart of (1.3) is

$$
\Delta_{\Sigma} u=n H\left\langle N, \partial_{t}\right\rangle-2\left\langle\nabla^{\Sigma} \log \varrho, \nabla^{\Sigma} u\right\rangle
$$

where $\Delta_{\Sigma}$ is the Laplace-Beltrami operator in $\Sigma$. Indeed if $\nabla^{\Sigma}$ denotes the intrinsic covariant derivative in $\Sigma$, we have

$$
\nabla^{\Sigma} u=(\bar{\nabla} t)^{T}=\varrho^{-2} \partial_{t}^{T}
$$

where $T$ denotes tangential projection onto $T \Sigma$. Hence we obtain

$$
\Delta_{\Sigma} u=n H \varrho^{-2}\left\langle\partial_{t}, N\right\rangle+\left\langle\nabla^{\Sigma} \varrho^{-2}, \partial_{t}^{T}\right\rangle
$$

from where the formula (1.5) above follows.

In particular, minimal graphs in $N=M \times_{\varrho} \mathbb{R}$ have height function that satisfies the weighted harmonic equation

$$
\Delta_{\Sigma} u+2\left\langle\nabla^{\Sigma} \log \varrho, \nabla^{\Sigma} u\right\rangle=0 .
$$

This may be considered as a PDE in $\Omega$ if we replace the metric $g$ by the induced metric with components given by (1.2).

Denoting

$$
\sigma^{i j}=g^{i j}-\frac{u^{i} u^{j}}{W^{2}}
$$

we can write (1.3) in non-divergence form as

$$
\sigma^{i j} u_{i ; j}+(\log \varrho)^{i} u_{i}\left(1+\frac{1}{\varrho^{2} W^{2}}\right)=n H W .
$$

\section{Main results}

The existence of Killing graphs with prescribed mean curvature $H$ over bounded domains $\Omega \subset M$ with continuous boundary data on $\partial \Omega$ was established in [11, Theorem 2] under suitable conditions on the Ricci curvature on $\Omega$, the mean curvature function $H$, and on the mean curvature of the Killing cylinder over $\partial \Omega$; see also [10].

In this paper we mainly focus on the setting where $M$ is a Cartan-Hadamard manifold with sectional curvatures controlled from above and below by some radial functions. We prove quantitative a priori height and gradient estimates for solutions of (1.3) on geodesic balls $\Omega=B(o, k) \subset M$ under natural conditions on the prescribed mean curvature function in terms of sectional curvatures $K_{M}$ and the warping function $\varrho$. These estimates allow us to use the continuity method (the Leray-Schauder method) and hence are enough to guarantee the existence of solutions to the following Dirichlet problem

$$
\begin{cases}\operatorname{div}\left(\frac{\nabla u}{W}\right)+\left\langle\nabla \log \varrho, \frac{\nabla u}{W}\right\rangle=n H & \text { in } \Omega \\ u \mid \partial \Omega=\varphi & \text { in } \partial \Omega\end{cases}
$$

where $\varphi \in C(\partial \Omega)$. We formulate the (local) existence result in geodesic balls on CartanHadamard manifolds. 
Theorem 2.1 Let $M$ be a Cartan-Hadamard manifold, $\Omega=B(o, k) \subset M$, and $\varphi \in C(\partial \Omega)$. Suppose that the prescribed mean curvature function $H \in C^{\alpha}(\Omega)$ satisfies

$$
|H(x)|<H_{k-d(x)}
$$

in $\bar{\Omega}$, where $d(x)=\operatorname{dist}(x, \partial B(o, k))=k-r(x)$ and $H_{k-d}$ is the mean curvature of the Killing cylinder $\mathcal{C}_{k-d}$ over the geodesic sphere $\partial B(o, k-d)$. Then there exists a unique solution $u \in C^{2, \alpha}(\Omega) \cap C(\bar{\Omega})$ to $(2.1)$.

Above and in what follows we denote by $r(x)=d(x, o)$ the distance from $x$ to a fixed point $o \in M$. We notice that the mean curvature of the Killing cylinder $\mathcal{C}_{r}$ over a geodesic sphere $\partial B(o, r)$ is given by

$$
H_{r}=\frac{1}{n}\left(\Delta r+\frac{1}{\varrho}\langle\nabla \varrho, \nabla r\rangle\right)
$$

and therefore can be estimated from below in terms of a suitable model manifold $M_{-a^{2}(r)} \times_{\varrho_{+}}$ $\mathbb{R}$, where $M_{-a^{2}(r)}$ is a rotationally symmetric Cartan-Hadamard manifold with radial sectional curvatures equal to $-a^{2}(r)$ and $\varrho_{+}: M \rightarrow(0, \infty)$ is a positive rotationally symmetric $C^{1}$ function such that

$$
\frac{1}{\varrho}\langle\nabla \varrho, \nabla r\rangle=\frac{\partial_{r} \varrho}{\varrho} \geq \frac{\partial_{r} \varrho_{+}}{\varrho_{+}} .
$$

To formulate the next corollary and for later purposes we denote by $f_{\kappa} \in C^{\infty}([0, \infty))$ the solution of the Jacobi equation

$$
\left\{\begin{array}{l}
f_{\kappa}^{\prime \prime}-\kappa^{2} f_{\kappa}=0 \\
f_{\kappa}(0)=0 \\
f_{\kappa}^{\prime}(0)=1
\end{array}\right.
$$

whenever $\kappa:[0, \infty) \rightarrow[0, \infty)$ is a smooth function.

Corollary 2.2 Let M be a Cartan-Hadamard manifold whose radial sectional curvatures are bounded from above by

$$
K\left(P_{x}\right) \leq-a(r(x))^{2}
$$

for some smooth function $a:[0, \infty) \rightarrow[0, \infty)$. Suppose, moreover, that $(2.2)$ holds with some positive rotationally symmetric $C^{1}$ function $\varrho_{+}=\varrho_{+}(r)$. If the prescribed mean curvature function $H \in C^{\alpha}(\Omega), \Omega=B(o, k)$, satisfies

$$
n|H(x)|<\frac{(n-1) f_{a}^{\prime}(r(x))}{f_{a}(r(x))}+\frac{\varrho_{+}^{\prime}(r(x))}{\varrho_{+}(r(x))}
$$

for all $x \in \bar{\Omega}$, then there exists a unique solution $u \in C^{2, \alpha}(\Omega) \cap C(\bar{\Omega})$ to (2.1).

As mentioned above the proofs of Theorem 2.1 and Corollary 2.2 for boundary data $\varphi \in C^{2, \alpha}(\partial \Omega)$ follow from the well-known continuity method once the a priori height and gradient estimates are at our disposal. The case of a continuous boundary values $\varphi \in C(\partial \Omega)$ can be treated as in [11]; see also [4].

Our main object in this paper is the asymptotic Dirichlet problem for Killing graphs with prescribed mean curvature and behaviour at infinity. To solve the problem, we extend the given boundary value function $\varphi \in C\left(\partial_{\infty} M\right)$ to a continuous function $\varphi \in C(\bar{M})$; see Sect. 5 
for the notation. Then we apply Corollary 2.2 for an exhaustion $\Omega_{k}=B(o, k), k \in \mathbb{N}$, of $M$ to obtain a sequence of solutions $u_{k}$ with boundary values $u_{k} \mid \partial \Omega_{k}=\varphi$. Under a suitable bound on $|H|$ in terms of a comparison manifold $M_{-a^{2}(r)} \times \varrho_{\varrho_{+}} \mathbb{R}$ we obtain a global height estimate and, consequently together with Schauder estimates, the sequence is uniformly bounded in the $C^{2, \alpha}$-norm. Hence there exists a subsequence that converges in the $C^{2, \alpha}$-norm to a global solution $u$ to the equation

$$
\operatorname{div}\left(\frac{\nabla u}{W}\right)+\left\langle\nabla \log \varrho, \frac{\nabla u}{W}\right\rangle=n H
$$

in $M$. Finally, under suitable curvature upper and lower bounds as well as conditions on $|H|$ we are able to construct (local) barriers at infinity and prove that the solution $u$ extends continuously to $\partial_{\infty} M$ and attains the given boundary values $\varphi$ there.

The following two solvability theorems will be proven in Sect. 6 .

Theorem 2.3 Let $M$ be a Cartan-Hadamard manifold satisfying the curvature assumptions (5.1) and (A1)-(A7) in Sect. 5. Furthermore, assume that the prescribed mean curvature function $H: M \rightarrow \mathbb{R}$ satisfies the assumptions (4.18) and (5.7) with a convex warping function $\varrho$ satisfying (4.13), (4.14), (5.8), and (5.9). Then there exists a unique solution $u: M \rightarrow \mathbb{R}$ to the Dirichlet problem

$$
\left\{\begin{array}{l}
\operatorname{div}_{-} \log \varrho \frac{\nabla u}{\sqrt{\varrho^{-2}+|\nabla u|^{2}}}=n H(x) \text { in } M \\
u \mid \partial_{\infty} M=\varphi
\end{array}\right.
$$

for any continuous function $\varphi: \partial_{\infty} M \rightarrow \mathbb{R}$.

Theorem 2.4 Let $M$ be a Cartan-Hadamard manifold satisfying the curvature assumptions (5.1) and (A1)-(A7) in Section 5. Furthermore, assume that the prescribed mean curvature function $H: M \rightarrow \mathbb{R}$ satisfies the assumptions (4.25) and (5.7) with a convex warping function $\varrho$ satisfying (4.19), (5.8), and (5.9). Then there exists a unique solution $u: M \rightarrow \mathbb{R}$ to the Dirichlet problem (2.4) for any continuous function $\varphi: \partial_{\infty} M \rightarrow \mathbb{R}$.

Remark 2.5 The following example illustrates the need of our assumptions about the warping function $\varrho$ in Theorems 2.3 and 2.4. Let $N$ be the $(n+1)$-dimensional hyperbolic space $\mathbb{H}^{n+1}$ and consider the Killing vector field $X$ in $\mathbb{H}^{n+1}$ corresponding to a one-parameter family of parabolic isometries of $\mathbb{H}^{n+1}$ preserving a given ideal point, say $p_{0} \in \partial_{\infty} \mathbb{H}^{n+1}$. This configuration cannot be directly compared with a rotationally invariant model (that is, invariant by a one-parameter family of elliptic isometries) as we have assumed for instance in conditions 4.13 and 4.14. This borderline case of a one-parameter family of parabolic isometries and the corresponding Killing field in $\mathbb{H}^{n+1}$ were studied by Ripoll and Telichevsky in [17] using different techniques relying on a variant of the Perron method.

\section{A priori height and gradient estimates}

Throughout this section we denote by $\Omega_{k}=B(o, k)$ the geodesic ball centered at a given point $o \in M$ with radius $k \in \mathbb{N}$, and by $d(\cdot)=\operatorname{dist}\left(\cdot, \partial \Omega_{k}\right)$ the distance function to the boundary of $\Omega_{k}$. 


\subsection{Height estimate}

Fix $k \in \mathbb{N}$ and suppose that $u_{k} \in C^{2}\left(\Omega_{k}\right)$ is a solution of the Dirichlet problem (2.1). We aim to show that the function

$$
v_{k}(x)=\sup _{\partial \Omega_{k}} \varphi_{k}+h(d(x))
$$

where $h$ will be determined later, is an upper barrier for the solution $u_{k}$. It suffices to show (see [19, p. 795] or [10, pp. 239-240]) that $v_{k}$ is a barrier in an open neighbourhood of $\partial \Omega_{k}$ in which the points can be joined to $\partial \Omega_{k}$ by unique geodesics. In this neighbourhood the distance function $d$ has the same regularity as $\partial \Omega_{k}$ and therefore the derivatives of $d$ in the following computations are well-defined.

Since $X$ is Killing field, we have

$$
\langle\nabla \log \varrho, \nabla d\rangle=\frac{1}{\varrho}\langle\nabla \varrho, \nabla d\rangle=-\frac{1}{\varrho^{2}}\left\langle\bar{\nabla}_{X} X, \nabla d\right\rangle=:-\kappa(d),
$$

where $\kappa$ is the principal curvature of the Killing cylinder $\mathcal{C}_{k-d}$ over the geodesic sphere $\partial B(o, k-d)$. This implies that

$$
\begin{aligned}
\mathcal{Q}\left[v_{k}\right] & =\operatorname{div}\left(\frac{h^{\prime} \nabla d}{\sqrt{\varrho^{-2}+h^{\prime 2}}}\right)-\kappa \frac{h^{\prime}}{\sqrt{\varrho^{-2}+h^{\prime 2}}} \\
& =\frac{h^{\prime}}{\sqrt{\varrho^{-2}+h^{\prime 2}}}(\Delta d-\kappa)+\partial_{d}\left(\frac{h^{\prime}}{\sqrt{\varrho^{-2}+h^{\prime 2}}}\right),
\end{aligned}
$$

where $\partial_{d}$ denotes the derivative to the direction $\nabla d$. However,

$$
\Delta d-\kappa=-n H_{k-d},
$$

where $H_{k-d}$ is the mean curvature of the cylinder $\mathcal{C}_{k-d}$, and we have

$$
\begin{aligned}
\partial_{d}\left(\frac{h^{\prime}}{\sqrt{\varrho^{-2}+h^{\prime 2}}}\right) & =\frac{h^{\prime \prime}}{\sqrt{\varrho^{-2}+h^{\prime 2}}}-\frac{h^{\prime}}{\left(\varrho^{-2}+h^{\prime 2}\right)^{3 / 2}}\left(\varrho^{-2} \kappa+h^{\prime} h^{\prime \prime}\right) \\
& =\frac{\varrho^{-2}}{\left(\varrho^{-2}+h^{\prime 2}\right)^{3 / 2}}\left(h^{\prime \prime}-\kappa h^{\prime}\right) .
\end{aligned}
$$

Hence it follows that

$$
\mathcal{Q}\left[v_{k}\right]=-\frac{h^{\prime}}{\sqrt{\varrho^{-2}+h^{\prime 2}}} n H_{k-d}+\frac{\varrho^{-2}}{\left(\varrho^{-2}+h^{\prime 2}\right)^{3 / 2}}\left(h^{\prime \prime}-\kappa h^{\prime}\right) .
$$

Suppose that the principal curvature of the Killing cylinder $\mathcal{C}_{k-d}$ satisfies

$$
\kappa(d) \geq-\frac{\varrho_{0}^{\prime}(d)}{\varrho_{0}(d)},
$$

where $\varrho_{0}$ is a smooth positive increasing function on $[0, \infty)$. We note already at this point that, in the case of Cartan-Hadamard manifolds, $\nabla d=-\nabla r$ and this agrees with the assumption (4.13). Then define the function $h$ as

$$
h(d)=C \int_{0}^{d} \varrho_{0}^{-1}(t) \mathrm{d} t
$$


for some constant $C>0$ to be fixed later. Now, since $h^{\prime}>0$, we have

$$
h^{\prime \prime}-\kappa h^{\prime} \leq 0
$$

and

$$
\mathcal{Q}\left[v_{k}\right] \leq-\frac{h^{\prime}}{\sqrt{\varrho^{-2}+h^{\prime 2}}} n H_{k-d}=-\frac{C \varrho}{\sqrt{\varrho_{0}^{2}+C^{2} \varrho^{2}}} n H_{k-d} .
$$

Assuming that

$$
|H|<H_{k-d}
$$

in $\bar{\Omega}_{k}$ and choosing the constant $C$ as

$$
C^{2}>\frac{H^{2} / H_{k-d}^{2}}{1-H^{2} / H_{k-d}^{2}} \frac{\sup \varrho_{0}^{2}}{\inf \varrho^{2}}
$$

we see that

$$
\mathcal{Q}\left[v_{k}\right]-n H \leq 0
$$

and hence $v_{k}$ is an upper barrier for $u_{k}$.

Similarly we see that the function

$$
v_{k}^{-}=\inf _{\partial \Omega_{k}} \varphi_{k}-h(d)
$$

is a lower barrier for $u_{k}$ and together these barriers give the following height estimate.

Lemma 3.1 Assume that

$$
|H|<H_{k-d}
$$

in $\bar{\Omega}_{k}$ and that $u_{k}$ is a solution to the Dirichlet problem (2.1). Then there exists a constant $C=C\left(\Omega_{k}\right)$ such that

$$
\sup _{\Omega_{k}}\left|u_{k}\right| \leq C+\sup _{\partial \Omega_{k}}|\varphi|
$$

\subsection{Boundary gradient estimate}

For given $\varepsilon>0$ we define an annulus

$$
U_{k}(\varepsilon)=\left\{x \in \Omega_{k}: d(x)<\varepsilon\right\} .
$$

In order to obtain a boundary gradient estimate, we aim to show that a function of the form

$$
w(x)=g(d(x))+\psi(x)
$$

is an upper barrier in the set $U_{k}(\varepsilon)$ for a fixed $\varepsilon \in(0,1 / 2)$ chosen so that $d$ is smooth in $U_{k}(\varepsilon)$. Here we denote by $\psi$ the extension of the boundary data that is constant along geodesics issuing perpendicularly from $\partial \Omega_{k}$, i.e. $\psi\left(\exp _{y} t \nabla d(y)\right)=\varphi(y)$, where $y \in \partial \Omega_{k}$ and $\nabla d(y)$ is the unit inward normal to $\partial \Omega_{k}$ at $y$. From (1.7) we have that

$$
\mathcal{Q}[w]=\frac{1}{W} \Delta w-\frac{1}{W^{3}}\left\langle\nabla_{\nabla w} \nabla w, \nabla w\right\rangle-\frac{1}{\varrho^{2}}\left(1+\frac{1}{\varrho^{2} W^{2}}\right)\left\langle\bar{\nabla}_{X} X, \frac{\nabla w}{W}\right\rangle,
$$


where

$$
W=\sqrt{\varrho^{-2}+|\nabla w|^{2}}=\sqrt{\varrho^{-2}+g^{\prime 2}+|\nabla \psi|^{2}} .
$$

Since

$$
\nabla w=g^{\prime} \nabla d+\nabla \psi,
$$

with $\langle\nabla d, \nabla \psi\rangle=0$, it follows that

$$
\Delta w=g^{\prime} \Delta d+g^{\prime \prime}+\Delta \psi
$$

and

$$
\left\langle\nabla_{\nabla w} \nabla w, \nabla w\right\rangle=g^{\prime 2} g^{\prime \prime}-g^{\prime}\left\langle\nabla_{\nabla \psi} \nabla d, \nabla \psi\right\rangle+\left\langle\nabla_{\nabla \psi} \nabla \psi, \nabla \psi\right\rangle .
$$

Moreover, by (3.2)

$$
\left\langle\nabla w, \bar{\nabla}_{X} X\right\rangle=g^{\prime}\left\langle\nabla d, \bar{\nabla}_{X} X\right\rangle+\left\langle\nabla \psi, \bar{\nabla}_{X} X\right\rangle=g^{\prime} \varrho^{2} \kappa+\left\langle\nabla \psi, \bar{\nabla}_{X} X\right\rangle .
$$

Using the expression (3.5) we obtain that

$$
\begin{aligned}
\mathcal{Q}[w]= & \frac{1}{W}\left(g^{\prime \prime}+g^{\prime} \Delta d+\Delta \psi\right)-\frac{1}{W^{3}}\left(g^{\prime 2} g^{\prime \prime}-g^{\prime}\left\langle\nabla_{\nabla \psi} \nabla d, \nabla \psi\right\rangle+\left\langle\nabla_{\nabla \psi} \nabla \psi, \nabla \psi\right\rangle\right) \\
& -\frac{1}{W}\left(1+\frac{1}{\varrho^{2} W^{2}}\right)\left(g^{\prime} \kappa+\left\langle\bar{\nabla}_{\frac{X}{|X|}} \frac{X}{|X|}, \nabla \psi\right\rangle\right)
\end{aligned}
$$

and combining with the previous reasoning, this results to

$$
\begin{aligned}
\mathcal{Q}[w]= & \frac{g^{\prime \prime}}{W^{3}}\left(\varrho^{-2}+|\nabla \psi|^{2}\right)+\frac{g^{\prime}}{W}\left(\Delta d-\left(1+\frac{1}{\varrho^{2} W^{2}}\right) \kappa\right)+\frac{1}{W} \Delta \psi \\
& -\frac{1}{W^{3}}\left\langle\nabla_{\nabla \psi} \nabla \psi, \nabla \psi\right\rangle+\frac{g^{\prime}}{W^{3}}\left\langle\nabla_{\nabla \psi} \nabla d, \nabla \psi\right\rangle \\
& -\frac{1}{W}\left(1+\frac{1}{\varrho^{2} W^{2}}\right)\left\langle\bar{\nabla}_{\frac{X}{|X|}} \frac{X}{|X|}, \nabla \psi\right\rangle .
\end{aligned}
$$

We note that

$$
\frac{1}{W} \Delta \psi-\frac{1}{W^{3}}\left\langle\nabla_{\nabla \psi} \nabla \psi, \nabla \psi\right\rangle=\frac{1}{W}\left(g^{i j}-\frac{\psi^{i} \psi^{j}}{W^{2}}\right) \psi_{i ; j}
$$

and, on the other hand,

$$
\begin{aligned}
\frac{1}{W}\left(g^{i j}-\frac{\left(g^{\prime} d^{i}+\psi^{i}\right)\left(g^{\prime} d^{j}+\psi^{j}\right)}{W^{2}}\right) \psi_{i ; j} & =\frac{1}{W}\left(g^{i j}-\frac{\psi^{i} \psi^{j}}{W^{2}}\right) \psi_{i ; j}-\frac{2 g^{\prime} d^{i} \psi^{j}}{W^{3}} \psi_{i ; j} \\
& =\frac{1}{W}\left(g^{i j}-\frac{\psi^{i} \psi^{j}}{W^{2}}\right) \psi_{i ; j}-\frac{2 g^{\prime}}{W^{3}}\left\langle\nabla_{\nabla d} \nabla \psi, \nabla \psi\right\rangle
\end{aligned}
$$

Moreover, the matrix $\left(\sigma^{i j}\right)$ has eigenvalues $1 /\left(\varrho^{2} W^{3}\right)$ and $1 / W$ which can be estimated as

$$
\max \left(\frac{1}{\varrho^{2} W^{3}}, \frac{1}{W}\right) \leq \frac{1}{\varrho^{2} W^{3}}+\frac{1}{W} \leq \frac{1}{W}\left(1+\varrho^{2}\right) .
$$


When $\varrho \geq 1$, this is trivial, and when $\varrho<1$ we can choose the constant $K$ in the definition (3.7) of $g$ such that this holds. Therefore we are able to estimate

$$
\begin{aligned}
\mathcal{Q}[w] \leq & \frac{g^{\prime \prime}}{W^{3}}\left(\varrho^{-2}+|\nabla \psi|^{2}\right)+\frac{g^{\prime}}{W}\left(\Delta d-\left(1+\frac{1}{\varrho^{2} W^{2}}\right) \kappa\right)+\frac{1}{W}\left(1+\varrho^{2}\right)\left\|\nabla^{2} \psi\right\| \\
& +\frac{3 g^{\prime}}{W^{3}}\langle\nabla \nabla \psi \nabla d, \nabla \psi\rangle+\frac{1}{W}\left(1+\frac{1}{\varrho^{2} W^{2}}\right)\langle\nabla \log \varrho, \nabla \psi\rangle \\
\leq & \frac{g^{\prime \prime}}{W^{3}}\left(\varrho^{-2}+|\nabla \psi|^{2}\right)-\frac{g^{\prime}}{W}\left(n H_{k-d}+\frac{1}{\varrho^{2} W^{2}} \kappa\right)+\frac{1}{W}\left(1+\varrho^{2}\right)\left\|\nabla^{2} \psi\right\| \\
& +\frac{3 g^{\prime}}{W^{3}}\left|I I_{k-d}(\nabla \psi, \nabla \psi)\right|+\frac{1}{W}\left(1+\frac{1}{\varrho^{2} W^{2}}\right)\langle\nabla \log \varrho, \nabla \psi\rangle .
\end{aligned}
$$

Now we choose

$$
g(d)=\frac{C}{\log (1+K)} \log (1+K d)
$$

where

$$
C=K(1+K \varepsilon) \log (1+K)
$$

and $K \geq(1-2 \varepsilon) \varepsilon^{-2}$ so large that

$$
C \geq 2\left(\max _{\bar{\Omega}_{k}}\left|u_{k}\right|+\max _{\bar{\Omega}_{k}}|\psi|\right) .
$$

Note that this choice yields $g \geq u_{k}$ on the "inner" boundary $\left\{x \in \Omega_{k}: d(x)=\varepsilon\right\}$ of $U_{k}(\varepsilon)$. Then, for $K$ large, we have

$$
1 \geq \frac{g^{\prime 2}}{W^{2}} \geq \frac{K^{4}(1+K \varepsilon)^{2}}{(1+K d)^{2} L+K^{4}(1+K \varepsilon)^{2}}=\frac{(1+K \varepsilon)^{2}}{\frac{(1+K d)^{2}}{K^{4}} L+(1+K \varepsilon)^{2}} \geq c_{1}^{2}>0,
$$

where

$$
c_{1}^{2} \leq \frac{(1+K \varepsilon)^{2}}{(1+K \varepsilon)^{2}+L}
$$

with

$$
L=\sup _{\Omega}\left(\varrho^{-2}+|\nabla \psi|^{2}\right) .
$$

We also have

$$
g^{\prime \prime}(d)=-\frac{g^{\prime 2}}{K(1+K \varepsilon)},
$$

which implies that

$$
\frac{g^{\prime \prime}}{W^{2}}=-\frac{1}{K(1+K \varepsilon)} \frac{g^{\prime 2}}{W^{2}} \leq-\frac{1}{K(1+K \varepsilon)} c_{1}^{2} .
$$

Hence we obtain

$$
\begin{aligned}
& \mathcal{Q}[w] \leq-\frac{1}{K(1+K \varepsilon)} c_{1}^{2} \frac{1}{W}\left(\varrho^{-2}+|\nabla \psi|^{2}\right)-c_{1}\left(n H_{k-d}+\frac{1}{\varrho^{2} W^{2}} \kappa\right) \\
& +\frac{1}{W}\left(1+\varrho^{2}\right)|| \nabla^{2} \psi\left\|+\frac{g^{\prime}}{W} \frac{|\nabla \psi|^{2}}{W^{2}}\right\| I I_{k-d}||+\frac{|\nabla \psi|}{W}|\nabla \log \varrho|\left(1+\frac{1}{\varrho^{2} W^{2}}\right) .
\end{aligned}
$$


However,

$$
\frac{1}{\varrho^{2} W^{2}} \geq \frac{c_{1}^{2}}{\varrho^{2} g^{\prime 2}} \geq\left(\frac{1+K d}{K^{2}(1+K \varepsilon)}\right)^{2} \frac{c_{1}^{2}}{\varrho^{2}} \geq \frac{1}{K^{4}(1+K \varepsilon)^{2}} \frac{c_{1}^{2}}{\varrho^{2}}
$$

and

$$
\frac{1}{W} \geq \frac{1+K d}{K^{2}(1+K \varepsilon)} c_{1} \geq \frac{1}{K^{2}(1+K \varepsilon)} c_{1} .
$$

Combining these with the fact that $W \geq K^{2}$, we obtain the estimate

$$
\begin{aligned}
\mathcal{Q}[w] \leq & -c_{1} \frac{K+\kappa}{K^{4}(1+K \varepsilon)^{2}} \frac{c_{1}^{2}}{\varrho^{2}}-c_{1} n H_{k-d}+\frac{1}{W}\left(1+\varrho^{2}\right)\left\|\nabla^{2} \psi\right\| \\
& +\frac{g^{\prime}}{W} \frac{|\nabla \psi|^{2}}{W^{2}}|| I I_{k-d} \|+\left(1+\frac{1}{\varrho^{2}} \frac{1}{K^{2}}\right) \frac{|\nabla \psi|}{W}|\nabla \log \varrho| \\
\leq & -c_{1} \frac{K+\kappa}{K^{4}(1+K \varepsilon)^{2}} \frac{c_{1}^{2}}{\varrho^{2}}-c_{1} n H_{k-d}+\frac{1}{K^{2}}\left(1+\varrho^{2}\right)\left\|\nabla^{2} \psi\right\| \\
& +|\nabla \psi|^{2}|| I I_{k-d} \| \frac{1}{K^{4}}+\left(1+\frac{1}{\varrho^{2} K^{4}}\right)|\nabla \psi||\nabla \log \varrho| \frac{1}{K^{2}} .
\end{aligned}
$$

Therefore

$$
\begin{aligned}
& \mathcal{Q}[w]-n H \leq-n\left(c_{1} H_{k-d}+H\right)-c_{1} \frac{K+\kappa}{K^{4}(1+K \varepsilon)^{2}} \frac{c_{1}^{2}}{\varrho^{2}}+\frac{1}{K^{2}}\left(1+\varrho^{2}\right)\left\|\nabla^{2} \psi\right\| \\
& +|\nabla \psi|^{2}|| I I_{k-d}\left\|\frac{1}{K^{4}}+\left(1+\frac{1}{\varrho^{2} K^{4}}\right)|\nabla \psi \| \nabla \log \varrho| \frac{1}{K^{2}} .\right.
\end{aligned}
$$

Finally observe that

$$
c_{1} H_{k-d} \geq|H|
$$

if we choose $K$ such that

$$
\frac{H^{2}}{H_{k-d}^{2}} \leq c_{1}^{2} \leq \frac{(1+K \varepsilon)^{2}}{(1+K \varepsilon)^{2}+L}
$$

that is

$$
(1+K \varepsilon)^{2} \geq L \frac{H^{2} / H_{k-d}^{2}}{1-H^{2} / H_{k-d}^{2}} .
$$

Taking (3.6), (3.8), (3.9) and (3.10) into account, we can choose

$$
K=K\left(\Omega_{k}, H,\|\psi\|_{C^{2}}, \varepsilon, \sup _{\Omega_{k}}|u|\right)
$$

so large that

$$
\mathcal{Q}[w]-n H \leq 0
$$

holds in $U_{k}(\varepsilon)$. This suffices for the following boundary gradient estimate. 
Lemma 3.2 Assume that

$$
|H|<H_{k-d}
$$

in $\bar{\Omega}_{k}$ and that $u_{k}$ is a solution to the Dirichlet problem (2.1). Then there exists a constant

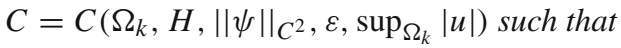

$$
\max _{\partial \Omega_{k}}\left|\nabla u_{k}\right| \leq C
$$

\subsection{Interior gradient estimate}

In this subsection we prove a quantitative interior gradient estimate that is interesting on its own. The proof is based on the technique due to Korevaar and Simon [14], and further developed by Wang [20]. We will perform the computations in a coordinate free way.

Let $u$ be a ( $C^{3}$-smooth) positive solution of the Eq. (1.3) in a ball $B(p, R) \subset M$. Suppose that sectional curvatures in $B(p, R)$ are bounded from below by $-K_{0}^{2}$ for some constant $K_{0}=K_{0}(p, R) \geq 0$. We consider a nonnegative and smooth function $\eta$ with $\eta=0$ in $M \backslash B(p, R)$ and define a function $\chi$ in $B(p, R)$ of the form

$$
\chi=\eta \gamma(u) \psi\left(|\nabla u|^{2}\right)
$$

where the functions $\eta, \gamma$ and $\psi$ will be specified later.

Suppose that $\chi$ attains its maximum at $x_{0} \in B(p, R)$, and without loss of generality, that $\eta\left(x_{0}\right) \neq 0$. Then at $x_{0}$

$$
(\log \chi)_{j}=\frac{\eta_{j}}{\eta}+\frac{\gamma^{\prime}}{\gamma} u_{j}+2 \frac{\psi^{\prime}}{\psi} u^{k} u_{k ; j}=0
$$

and therefore

$$
2 \frac{\psi^{\prime}}{\psi} u^{k} u_{k ; j}=-\left(\frac{\eta_{j}}{\eta}+\frac{\gamma^{\prime}}{\gamma} u_{j}\right)
$$

Moreover, the matrix

$$
\begin{aligned}
(\log \chi)_{i ; j}= & (\log \eta)_{i ; j}+\left(\frac{\gamma^{\prime}}{\gamma}\right)^{\prime} u_{i} u_{j}+\frac{\gamma^{\prime}}{\gamma} u_{i ; j}+2 \frac{\psi^{\prime}}{\psi}\left(u^{k} u_{k ; i j}+u_{; i}^{k} u_{k ; j}\right) \\
& +4\left(\frac{\psi^{\prime}}{\psi}\right)^{\prime} u^{k} u_{k ; i} u^{\ell} u_{\ell ; j}
\end{aligned}
$$

is non-positive at $x_{0}$. Applying the Ricci identities for the Hessian of $u$ we have

$$
u_{k ; i j}=u_{i ; k j}=u_{i ; j k}+R_{k j i}^{\ell} u_{\ell},
$$

and this yields

$$
\begin{aligned}
(\log \chi)_{i ; j}= & \frac{\eta_{i ; j}}{\eta}+\frac{\gamma^{\prime \prime}}{\gamma} u_{i} u_{j}+\frac{\gamma^{\prime}}{\gamma} u_{i ; j}+\frac{\gamma^{\prime}}{\gamma}\left(\frac{\eta_{i}}{\eta} u_{j}+\frac{\eta_{j}}{\eta} u_{i}\right) \\
& +2 \frac{\psi^{\prime}}{\psi}\left(u^{k} u_{i ; j k}+u_{; i}^{k} u_{k ; j}\right)-2 \frac{\psi^{\prime}}{\psi} R_{j k i}^{\ell} u^{k} u_{\ell}+4\left(\left(\frac{\psi^{\prime}}{\psi}\right)^{\prime}-\frac{\psi^{\prime 2}}{\psi^{2}}\right) u^{k} u_{k ; i} u^{\ell} u_{\ell ; j}
\end{aligned}
$$

On the other hand, denoting

$$
f(x)=n H W-\langle\nabla \log \varrho, \nabla u\rangle\left(1+\frac{1}{\varrho^{2} W^{2}}\right),
$$


and differentiating both sides in (1.7) we have

$$
\sigma^{i j} u_{i ; j k}=f_{k}-\sigma_{; k}^{i j} u_{i ; j} .
$$

Contracting (3.14) with $u^{k}$, we get

$$
\begin{aligned}
\sigma^{i j} u^{k} u_{i ; j k}= & f_{k} u^{k}+\frac{1}{W^{2}} u^{k}\left(u_{; k}^{i} u^{j}+u^{i} u_{; k}^{j}\right) u_{i ; j} \\
& -\frac{2}{W^{4}} u^{i} u^{j} u_{i ; j}\left(-\varrho^{-2}(\log \varrho)_{k} u^{k}+u^{k} u^{\ell} u_{\ell ; k}\right) .
\end{aligned}
$$

Using the previous identity, (3.13) and noticing that

$$
\sigma^{i j} R_{j k i}^{\ell} u^{k} u_{\ell}=-\operatorname{Ric}_{g}(\nabla u, \nabla u),
$$

lengthy computations give

$$
\begin{aligned}
0 \geq & \sigma^{i j}(\log \chi)_{i ; j}=2 n \frac{\psi^{\prime}}{\psi}\langle\nabla H, \nabla u\rangle W+n H \frac{\gamma^{\prime}}{\gamma} \frac{1}{\varrho^{2} W}-n H \frac{1}{W}\left\langle\frac{\nabla \eta}{\eta}, \nabla u\right\rangle \\
& -2 n H \frac{1}{\varrho^{2} W} \frac{\psi^{\prime}}{\psi}\langle\nabla \log \varrho, \nabla u\rangle+4\left(\left(\frac{\psi^{\prime}}{\psi}\right)^{\prime}-\frac{\psi^{\prime 2}}{\psi^{2}}+\frac{3}{2} \frac{\psi^{\prime}}{\psi} \frac{1}{W^{2}}\right) \sigma^{i \ell} u^{j} u^{k} u_{k ; i} u_{j ; \ell} \\
& +\sigma^{i j} \frac{\eta_{i ; j}}{\eta}+2 \frac{\gamma^{\prime}}{\gamma} \frac{1}{\varrho^{2} W^{2}}\left\langle\frac{\nabla \eta}{\eta}, \nabla u\right\rangle+2 \frac{\psi^{\prime}}{\psi}\left(\operatorname{Ric}_{g}(\nabla u, \nabla u)-\nabla^{2} \log \varrho(\nabla u, \nabla u)\right) \\
& +\frac{4|\nabla u|^{2}}{\varrho^{2} W^{4}} \frac{\psi^{\prime}}{\psi}\langle\nabla \log \varrho, \nabla u\rangle^{2}-\frac{2}{\varrho^{2} W^{4}}\langle\nabla \log \varrho, \nabla u\rangle\left\langle\frac{\nabla \eta}{\eta}+\frac{\gamma^{\prime}}{\gamma} \nabla u, \nabla u\right\rangle \\
& -2 \frac{\psi^{\prime}}{\psi} \frac{1}{\varrho^{2} W^{2}} \nabla^{2} \log \varrho(\nabla u, \nabla u)+\left\langle\nabla \log \varrho, \frac{\nabla \eta}{\eta}\right\rangle\left(1+\frac{1}{\varrho^{2} W^{2}}\right) \\
& -\frac{2}{\varrho^{2} W^{4}}\left\langle\frac{\nabla \eta}{\eta}+\frac{\gamma^{\prime}}{\gamma} \nabla u, \nabla u\right\rangle\langle\nabla \log \varrho, \nabla u\rangle+\frac{\gamma^{\prime \prime}}{\gamma} \sigma^{i j} u_{i} u_{j}+2 \frac{\psi^{\prime}}{\psi} \sigma^{i \ell} \sigma^{j k} u_{k ; i} u_{j ; \ell} .
\end{aligned}
$$

Notice that (3.13) yields to

$$
\begin{aligned}
4 \frac{\psi^{\prime 2}}{\psi^{2}} \sigma^{i \ell} u^{j} u^{k} u_{k ; i} u_{j ; \ell} & =\left|\frac{\nabla \eta}{\eta}+\frac{\gamma^{\prime}}{\gamma} \nabla u\right|_{\sigma}^{2} \geq \frac{1}{\varrho^{2} W^{2}}\left|\frac{\nabla \eta}{\eta}+\frac{\gamma^{\prime}}{\gamma} \nabla u\right|_{g}^{2} \\
& =\frac{|\nabla u|^{2}}{\varrho^{2} W^{2}}\left|\frac{\nabla \eta}{|\nabla u| \eta}+\frac{\gamma^{\prime}}{\gamma} \frac{\nabla u}{|\nabla u|}\right|_{g}^{2} .
\end{aligned}
$$

Plugging this into the previous estimate, we get

$$
\begin{aligned}
& \frac{\psi^{2}}{\psi^{\prime 2}}\left(\left(\frac{\psi^{\prime}}{\psi}\right)^{\prime}-\frac{\psi^{\prime 2}}{\psi^{2}}+\frac{3}{2} \frac{\psi^{\prime}}{\psi} \frac{1}{W^{2}}\right) \frac{|\nabla u|^{2}}{\varrho^{2} W^{2}}\left|\frac{\nabla \eta}{|\nabla u| \eta}+\frac{\gamma^{\prime}}{\gamma} \frac{\nabla u}{|\nabla u|}\right|_{g}^{2} \\
& \quad+\frac{\gamma^{\prime \prime}}{\gamma} \sigma^{i j} u_{i} u_{j}+2 \frac{\psi^{\prime}}{\psi} \sigma^{i \ell} \sigma^{j k} u_{k ; i} u_{j ; \ell} \\
& \leq 2 n \frac{\psi^{\prime}}{\psi}|\nabla H||\nabla u| W+2 n \frac{\psi^{\prime}}{\psi}|H| \frac{|\nabla u|}{W} \frac{|\nabla \log \varrho|}{\varrho^{2}} \\
& \quad-2 \frac{\psi^{\prime}}{\psi}\left(\operatorname{Ric}_{g}(\nabla u, \nabla u)-\nabla^{2} \log \varrho(\nabla u, \nabla u)\right)+4 \frac{\psi^{\prime}}{\psi} \frac{|\nabla \log \varrho|^{2}}{\varrho^{2}} \frac{|\nabla u|^{4}}{W^{4}} \\
& \quad+2 \frac{\psi^{\prime}}{\psi} \frac{\left|\nabla^{2} \log \varrho\right|}{\varrho^{2}} \frac{|\nabla u|^{2}}{W^{2}}+n|H| \frac{\gamma^{\prime}}{\gamma} \frac{1}{\varrho^{2} W}+n|H|\left|\frac{\nabla \eta}{\eta}\right| \frac{|\nabla u|}{W}-\sigma^{i j} \frac{\eta_{i ; j}}{\eta}
\end{aligned}
$$




$$
\begin{aligned}
& +2 \frac{\gamma^{\prime}}{\gamma} \frac{1}{\varrho^{2} W} \frac{|\nabla u|}{W}\left|\frac{\nabla \eta}{\eta}\right|+4 \frac{|\nabla \log \varrho|}{\varrho^{2}}\left(\left|\frac{\nabla \eta}{\eta}\right| \frac{|\nabla u|^{2}}{W^{4}}+\frac{\gamma^{\prime}}{\gamma} \frac{|\nabla u|^{3}}{W^{4}}\right) \\
& +|\nabla \log \varrho|\left|\frac{\nabla \eta}{\eta}\right|\left(1+\frac{1}{\varrho^{2} W^{2}}\right) .
\end{aligned}
$$

Suppose that $|\nabla u|\left(x_{0}\right)>1$. Otherwise we are done. Hence, following [20], we set

$$
\psi(t)=\log t
$$

where $t=|\nabla u|^{2}$. Then we have

$$
\frac{|\nabla u|^{2}}{W^{2}} \frac{\psi^{2}}{\psi^{\prime 2}}\left(\left(\frac{\psi^{\prime}}{\psi}\right)^{\prime}-\frac{\psi^{\prime 2}}{\psi^{2}}+\frac{3}{2} \frac{\psi^{\prime}}{\psi} \frac{1}{W^{2}}\right)=\frac{t}{W^{2}}\left(\log t \frac{\frac{1}{2} t-\varrho^{-2}}{t+\varrho^{-2}}-2\right) .
$$

Now we fix a constant

$$
\max \left\{\frac{2}{3}, \frac{\varrho^{2}}{1+\varrho^{2}}\right\}<\beta<1
$$

and suppose that

$$
\frac{t}{W^{2}}=\frac{|\nabla u|^{2}}{W^{2}} \geq \beta
$$

Setting $\frac{1}{\varrho^{2}} \frac{\beta}{1-\beta}=: e^{\delta^{\prime}}, \delta=\frac{3}{2} \beta-1$, and $\mu:=2 \beta \frac{\delta \delta^{\prime}-2}{\delta^{\prime}}$, we get

$$
\begin{aligned}
& \mu \log |\nabla u| \frac{1}{\varrho^{2}}\left|\frac{\nabla \eta}{|\nabla u| \eta}+\frac{\gamma^{\prime}}{\gamma} \frac{\nabla u}{|\nabla u|}\right|_{g}^{2}+\frac{\gamma^{\prime \prime}}{\gamma} \frac{|\nabla u|^{2}}{\varrho^{2} W^{2}} \\
& \quad+2 \frac{\psi^{\prime}}{\psi}|\nabla u|^{2}\left(\operatorname{Ric}_{g}\left(\frac{\nabla u}{|\nabla u|}, \frac{\nabla u}{|\nabla u|}\right)-\nabla^{2} \log \varrho\left(\frac{\nabla u}{|\nabla u|}, \frac{\nabla u}{|\nabla u|}\right)\right) \\
& \leq \frac{2}{\sqrt{\beta} \delta^{\prime}}\left(n|\nabla H|+(1-\beta) n|H||\nabla \log \varrho|+2(1-\beta)|\nabla \log \varrho|^{2}+(1-\beta)\left|\nabla^{2} \log \varrho\right|\right) \\
& \quad+\sqrt{1-\beta} \frac{1}{\varrho} \frac{\gamma^{\prime}}{\gamma}(n|H|+4|\nabla \log \varrho|)+2 \sqrt{1-\beta} \frac{1}{\varrho} \frac{\gamma^{\prime}}{\gamma}\left|\frac{\nabla \eta}{\eta}\right| \\
& \quad+\left|\frac{\nabla \eta}{\eta}\right|(n|H|+(6-5 \beta)|\nabla \log \varrho|)-\sigma^{i j} \frac{\eta_{i ; j}}{\eta} .
\end{aligned}
$$

By modifying the argument in [18, Proof of Theorem 4.1, Case 2] we may assume that the maximum point $x_{0}$ is not in the cut-locus $C(p)$ of $p$. Then we choose $\eta$ as

$$
\eta=\hat{\eta}^{2}
$$

where

$$
\hat{\eta}=1-\frac{1}{C_{R}} \int_{0}^{r} \xi(\tau) \mathrm{d} \tau, \quad r=d(\cdot, p),
$$

with

$$
C_{R}=\int_{0}^{R} \xi(\tau) \mathrm{d} \tau
$$

and $\xi(\tau)=K_{0}^{-1} \sinh \left(K_{0} \tau\right)$ if $K_{0}>0$ and $\xi(\tau)=\tau$ if $K_{0}=0$. Denoting

$$
\kappa=\varrho^{-2}\left\langle\bar{\nabla}_{X} \bar{\nabla} r, X\right\rangle=\langle\nabla r, \nabla \log \varrho\rangle,
$$


one can show that $|\nabla \eta|=2 \hat{\eta} \frac{\xi(r)}{C_{R}}$ and

$$
\begin{aligned}
& \Delta_{\Sigma} \eta=2 \hat{\eta} \Delta_{\Sigma} \hat{\eta}+2\left|\nabla^{\Sigma} \hat{\eta}\right|^{2} \\
& \leq 2 \hat{\eta}(r) \frac{\xi(r)}{C_{R}}\left|(n-1) \frac{\xi^{\prime}(r)}{\xi(r)}+\kappa+n\right| H|+(1-\beta)| \frac{\xi^{\prime}(r)}{\xi(r)}-\kappa||+2 \frac{\xi^{2}(r)}{C_{R}^{2}} .
\end{aligned}
$$

As in [20], we set

$$
\gamma(u)=1+\frac{1}{M}\left(\min _{\bar{B}(p, R)} \varrho\right) u
$$

where $M>0$ is a constant to be fixed later. Then $\gamma^{\prime \prime}=0$ and hence

$$
\begin{aligned}
& \mu \log |\nabla u| \frac{1}{\varrho^{2}}\left|\frac{\nabla \eta}{|\nabla u| \eta}+\frac{\gamma^{\prime}}{\gamma} \frac{\nabla u}{|\nabla u|}\right|_{g}^{2} \\
& \quad+2 \frac{\psi^{\prime}}{\psi}|\nabla u|^{2}\left(\operatorname{Ric}_{g}\left(\frac{\nabla u}{|\nabla u|}, \frac{\nabla u}{|\nabla u|}\right)-\nabla^{2} \log \varrho\left(\frac{\nabla u}{|\nabla u|}, \frac{\nabla u}{|\nabla u|}\right)\right) \leq \tilde{M} \frac{1}{M \eta},
\end{aligned}
$$

where

$$
\begin{aligned}
\tilde{M}= & \frac{2}{\sqrt{\beta} \delta^{\prime}}\left(n|\nabla H|+(1-\beta) n|H||\nabla \log \varrho|+2(1-\beta)|\nabla \log \varrho|^{2}\right. \\
& \left.+(1-\beta)\left|\nabla^{2} \log \varrho\right|\right) M \eta+\sqrt{1-\beta}(n|H|+4|\nabla \log \varrho|) \eta+4 \sqrt{1-\beta} \frac{\xi(r)}{C_{R}} \hat{\eta} \\
& +2 \frac{\xi(r)}{C_{R}}(n|H|+(6-5 \beta)|\nabla \log \varrho|) M \hat{\eta} \\
& +M\left(2 \hat{\eta}(r) \frac{\xi(r)}{C_{R}}\left|(n-1) \frac{\xi^{\prime}(r)}{\xi(r)}+\kappa+n\right| H|+(1-\beta)| \frac{\xi^{\prime}(r)}{\xi(r)}-\kappa||+2 \frac{\xi^{2}(r)}{C_{R}^{2}}\right) .
\end{aligned}
$$

Let $L=L(p, R) \geq 0$ be chosen in such a way that

$$
\operatorname{Ric}_{g}+\nabla^{2} \log \varrho \geq-L g
$$

in $B(p, R)$. Then we obtain

$$
\mu \log |\nabla u| \frac{1}{\varrho^{2}}\left|\frac{\nabla \eta}{|\nabla u| \eta}+\frac{\gamma^{\prime}}{\gamma} \frac{\nabla u}{|\nabla u|}\right|_{g}^{2}-2 L \frac{1}{\delta^{\prime}} \leq \tilde{M} \frac{1}{M \eta} .
$$

Set $M=\max _{\bar{B}(p, R)} u$. We consider first the case

$$
\left|\frac{\nabla \eta}{|\nabla u| \eta}\right| \leq \frac{\gamma^{\prime}}{2 \gamma}
$$

Then we have

$$
\eta \log |\nabla u| \leq \frac{4 \gamma^{2} \varrho^{2}}{\mu \min _{\bar{B}(p, R)} \varrho^{2}}\left(\tilde{M} M+2 L M^{2} \frac{\eta}{\delta^{\prime}}\right) .
$$

On the other hand, when

$$
\frac{\gamma^{\prime}}{2 \gamma} \leq\left|\frac{\nabla \eta}{|\nabla u| \eta}\right|
$$


we have

$$
\eta|\nabla u| \leq \frac{4 \gamma}{\gamma^{\prime}} \frac{\xi(r)}{C_{R}}
$$

which implies that

$$
\eta \log |\nabla u| \leq \frac{4 \gamma}{\gamma^{\prime}} \frac{\xi(r)}{C_{R}}
$$

Hence at $x_{0}$

$$
\eta \log |\nabla u| \leq \max \left\{\frac{4 \gamma\left(u\left(x_{0}\right)\right) \xi\left(r\left(x_{0}\right)\right)}{\gamma^{\prime}\left(u\left(x_{0}\right)\right) C_{R}}, \frac{4 \gamma^{2}\left(u\left(x_{0}\right)\right) \varrho^{2}\left(x_{0}\right)}{\mu \min _{\bar{B}(p, R)} \varrho^{2}}\left(\tilde{M} M+2 L M^{2} \frac{1}{\delta^{\prime}}\right)\right\} .
$$

Since $\eta(p)=1$ and $\gamma(p) \geq 1$ we conclude that

$$
\begin{aligned}
& \log |\nabla u(p)| \leq \eta(p) \gamma(p) \log |\nabla u(p)| \leq \eta\left(x_{0}\right) \gamma\left(x_{0}\right) \log \left|\nabla u\left(x_{0}\right)\right| \\
& \quad \leq \frac{4 M\left(1+\min _{\bar{B}(p, R)} \varrho\right)^{2}}{\min _{\bar{B}(p, R)} \varrho} \max \left\{\frac{\xi\left(r\left(x_{0}\right)\right)}{C_{R}}, \frac{\left(1+\min _{\bar{B}(p, R)} \varrho\right) \varrho^{2}\left(x_{0}\right)}{\mu \min _{\bar{B}(p, R)} \varrho}\left(\tilde{M}+2 L M \frac{1}{\delta^{\prime}}\right)\right\}
\end{aligned}
$$

unless $\left|\nabla u\left(x_{0}\right)\right| \leq 1$.

We have proven the following quantitative gradient estimate. Here we denote by $\mathcal{R}_{B}$ the Riemannian curvature tensor in a set $B$.

Lemma 3.3 Let $u$ be a positive solution of (1.3) in an open set $\Omega$ and let $B=B(p, R) \subset \Omega$. Then there exists a constant $C=C\left(\mathcal{R}_{B}, \varrho|B, H| B, u(p), \max _{\bar{B}} u, R\right)$ such that

$$
|\nabla u(p)| \leq C .
$$

If the gradient of $u$ is continuous up to the boundary of $\Omega$ and $\Omega$ is bounded, we obtain the following quantitative global estimate.

Lemma 3.4 Let $u$ be a positive solution of (1.3) in a bounded open set $\Omega$ and suppose, moreover, that $u \in C^{1}(\bar{\Omega})$. Then there exists a constant

$$
C=C\left(\mathcal{R}_{\Omega}, \varrho|\Omega, H| \Omega, u(p), \max _{\bar{\Omega}} u, \operatorname{diam}(\Omega), \max _{\partial \Omega}|\nabla u|\right)
$$

such that

$$
|\nabla u(p)| \leq C
$$

for every $p \in \bar{\Omega}$.

Proof Let $p \in \Omega$ and $R=\operatorname{diam}(\Omega)$. Define in $\bar{\Omega} \cap B(p, R)$ a function

$$
\chi=\eta \gamma(u) \psi\left(|\nabla u|^{2}\right)
$$

where $\eta, \gamma$, and $\psi$ are as in the previous proof. If $\chi$ attains its maximum in an interior point $x_{0} \in B(p, R) \cap \Omega$, the proof of Lemma 3.3 applies and we have a desired upper bound. Otherwise, $\chi$ attains its maximum at $x_{0} \in \partial \Omega$, but then $\left|\nabla u\left(x_{0}\right)\right| \leq \max _{\partial \Omega}|\nabla u|$ and again we are done.

We remark that a global gradient estimate for bounded Killing graphs follows immediately from (3.23), (3.20), and (3.21) in the case of bounded warping functions under some assumptions on the curvature. 
Corollary 3.5 Suppose that the sectional curvatures in $M$ satisfy $K_{M} \geq-K_{0}$ for some positive constant $K_{0}$. Suppose also that $\inf _{M} \varrho>0$ and that $\|\varrho\|_{C^{2}(M)}<+\infty$. If a function $u: M \rightarrow \mathbb{R}$ is uniformly bounded and the mean curvature of its graph satisfies $\|H\|_{C^{1}(M)}<$ $+\infty$ then the gradient of $u$ is uniformly bounded.

\section{Global barriers}

In this section we present two methods to obtain global (upper and lower) barriers for solutions to $(2.1)$.

In the case when $\widetilde{H}$ is constant along flow lines of $X$, that is, when $\widetilde{H}$ is a function in $M$, there is a conservation law (a flux formula) corresponding to the invariance of $\mathcal{A}_{\widetilde{H}}$ with respect to the flow generated by $X$. This flux formula for graphs is stated as

$$
\int_{\Gamma}\left\langle\frac{\nabla u}{W}, v\right\rangle \varrho \mathrm{d} \Gamma=\int_{\Omega} n \widetilde{H} \varrho \mathrm{d} M
$$

where $\Gamma=\partial \Omega$ and $v$ is the outward unit normal vector field along $\Gamma \subset M$.

Suppose for a while that $M$ is a model manifold with respect to a fixed pole $o \in M$ and that $\varrho=|X|$ is a radial function. In terms of polar coordinates $(r, \vartheta) \in \mathbb{R}^{+} \times \mathbb{S}^{n-1}$ centered at $o$ the metric in $M$ is of the form

$$
g=\mathrm{d} r^{2}+\xi^{2}(r) \mathrm{d} \vartheta^{2},
$$

where $\mathrm{d} \vartheta^{2}$ stands for the canonical metric in $\mathbb{S}^{n-1}$. Suppose that $\widetilde{H}$ and $u$ are also radial functions. Applying (4.1) to $\Omega=B(o, r)$, the geodesic ball centered at $o$ with radius $r$, we obtain

$$
\frac{u^{\prime}(r)}{\sqrt{\varrho^{-2}(r)+u^{\prime 2}(r)}} \varrho(r) \xi^{n-1}(r)=\int_{0}^{r} n \widetilde{H}(\tau) \varrho(\tau) \xi^{n-1}(\tau) \mathrm{d} \tau
$$

This is a first integral of (1.3) in this rotationally invariant setting. Indeed, taking derivatives on both sides of (4.2) with respect to $r$ we get

$$
n \tilde{H}(r)=\left(\frac{u^{\prime}(r)}{\sqrt{\varrho^{-2}(r)+u^{\prime 2}(r)}}\right)^{\prime}+\frac{u^{\prime}(r)}{\sqrt{\varrho^{-2}(r)+u^{\prime 2}(r)}}\left(\frac{\varrho^{\prime}(r)}{\varrho(r)}+(n-1) \frac{\xi^{\prime}(r)}{\xi(r)}\right) .
$$

On the other hand in this particular setting (1.3) becomes

$$
\begin{aligned}
n \tilde{H}(r)= & \operatorname{div}\left(\frac{u^{\prime}(r)}{\sqrt{\varrho^{-2}(r)+u^{\prime 2}(r)}} \partial_{r}\right)+\left(\frac{u^{\prime}(r)}{\sqrt{\varrho^{-2}(r)+u^{\prime 2}(r)}}\right) \frac{\varrho^{\prime}(r)}{\varrho(r)} \\
= & \left(\frac{u^{\prime}(r)}{\sqrt{\varrho^{-2}(r)+u^{\prime 2}(r)}}\right)^{\prime}+\frac{u^{\prime}(r)}{\sqrt{\varrho^{-2}(r)+u^{\prime 2}(r)}} \operatorname{div} \partial_{r} \\
& +\left(\frac{u^{\prime}(r)}{\sqrt{\varrho^{-2}(r)+u^{\prime 2}(r)}}\right) \frac{\varrho^{\prime}(r)}{\varrho(r)} \\
= & \left(\frac{u^{\prime}(r)}{\sqrt{\varrho^{-2}(r)+u^{\prime 2}(r)}}\right)^{\prime}+\frac{u^{\prime}(r)}{\sqrt{\varrho^{-2}(r)+u^{\prime 2}(r)}}\left((n-1) \frac{\xi^{\prime}(r)}{\xi(r)}+\frac{\varrho^{\prime}(r)}{\varrho(r)}\right) .
\end{aligned}
$$

It is convenient to write (4.2) in a "quadrature" form as follows

$$
u^{\prime 2}(r)=\frac{I^{2}(r) \varrho^{-2}(r)}{\varrho^{2}(r) \xi^{2(n-1)}(r)-I^{2}(r)},
$$


where

$$
I(r)=\int_{0}^{r} n \tilde{H}(\tau) \varrho(\tau) \xi^{n-1}(\tau) \mathrm{d} \tau .
$$

For instance, in the case when $\widetilde{H}$ is constant we have to impose a condition such as

$$
n|\tilde{H}| \leq \liminf _{r \rightarrow \infty} \frac{\varrho(r) \xi^{n-1}(r)}{\int_{0}^{r} \varrho(\tau) \xi^{n-1}(\tau) \mathrm{d} \tau}
$$

in order to guarantee the existence of radial solutions $u=u(r)$ to (1.3) for model manifolds. Note that the right-hand side in (4.4) is a sort of weighted isoperimetric ratio in $M$ with respect to the density $\varrho(r(x))=|X(x)|$. By l'Hospital's rule we see that (4.4) is equivalent to the requirement

$$
n|\tilde{H}| \leq \liminf _{r \rightarrow \infty}(n-1) \frac{\xi^{\prime}(r)}{\xi(r)}+\frac{\varrho^{\prime}(r)}{\varrho(r)} .
$$

This discussion motivates us to define in the general case a function of the form

$$
\begin{gathered}
u_{+}(x)=u_{+}(r(x)) \\
=\int_{r(x)}^{+\infty} \frac{\int_{0}^{\tau} n \widetilde{H}(s) \varrho_{+}(s) \xi_{+}^{n-1}(s) \mathrm{d} s}{\varrho_{+}(\tau) \sqrt{\varrho_{+}^{2}(\tau) \xi_{+}^{2(n-1)}(\tau)-\left(\int_{0}^{\tau} n \widetilde{H}(s) \varrho_{+}(s) \xi_{+}^{n-1}(s) \mathrm{d} s\right)^{2}}} \mathrm{~d} \tau \\
+\|\varphi\|_{C^{0}\left(\partial_{\infty} M\right)}
\end{gathered}
$$

for some nonnegative functions $\varrho_{+}(r(x)), \xi_{+}(r(x))$ and $\widetilde{H}(r(x))$ to be chosen later.

Plugging $u_{+}(x)=u_{+}(r(x))$ into the differential operator

$$
\mathcal{Q}[u]=\operatorname{div}\left(\frac{\nabla u}{W}\right)+\left\langle\nabla \log \varrho, \frac{\nabla u}{W}\right\rangle-n H
$$

yields

$$
\begin{aligned}
\mathcal{Q}\left[u_{+}\right]= & \left\langle\nabla \frac{u_{+}^{\prime}(r)}{\left(\varrho^{-2}(x)+u_{+}^{\prime 2}(r)\right)^{1 / 2}}, \partial_{r}\right\rangle \\
& +\frac{u_{+}^{\prime}(r)}{\left(\varrho^{-2}(x)+u_{+}^{\prime 2}(r)\right)^{1 / 2}}\left(\operatorname{div} \partial_{r}+\frac{1}{\varrho}\left\langle\nabla \varrho, \partial_{r}\right\rangle\right)-n H \\
= & \partial_{r}\left(\frac{u_{+}^{\prime}(r)}{\left(\varrho^{-2}(x)+u_{+}^{\prime 2}(r)\right)^{1 / 2}}\right)+\frac{u_{+}^{\prime}(r)}{\left(\varrho^{-2}(x)+u_{+}^{\prime 2}(r)\right)^{1 / 2}}\left(\Delta r+\frac{1}{\varrho}\left\langle\nabla \varrho, \partial_{r}\right\rangle\right)-n H \\
= & \partial_{r}\left(\frac{u_{+}^{\prime}(r)}{\left(\varrho_{+}^{-2}(r)+u_{+}^{\prime 2}(r)\right)^{1 / 2}} \frac{\left(\varrho_{+}^{-2}(r)+u_{+}^{\prime 2}(r)\right)^{1 / 2}}{\left(\varrho^{-2}(x)+u_{+}^{\prime 2}(r)\right)^{1 / 2}}\right) \\
& +\frac{u_{+}^{\prime}(r)}{\left(\varrho^{-2}(x)+u_{+}^{\prime 2}(r)\right)^{1 / 2}}\left(\Delta r+\frac{1}{\varrho}\left\langle\nabla \varrho, \partial_{r}\right\rangle\right)-n H \\
= & \frac{\left(\varrho_{+}^{-2}(r)+u_{+}^{\prime 2}(r)\right)^{1 / 2}}{\left(\varrho^{-2}(x)+u_{+}^{\prime 2}(r)\right)^{1 / 2}}\left[\frac{u_{+}^{\prime}(r)}{\left(\varrho_{+}^{-2}(r)+u_{+}^{\prime 2}(r)\right)^{1 / 2}}\left(\Delta r+\frac{1}{\varrho}\left\langle\nabla \varrho, \partial_{r}\right\rangle\right)\right.
\end{aligned}
$$




$$
\begin{aligned}
& \left.+\partial_{r}\left(\frac{u_{+}^{\prime}(r)}{\left(\varrho_{+}^{-2}(r)+u_{+}^{\prime 2}(r)\right)^{1 / 2}}\right)\right] \\
& +\frac{u_{+}^{\prime}(r)}{\left(\varrho_{+}^{-2}(r)+u_{+}^{\prime 2}(r)\right)^{1 / 2}} \partial_{r}\left(\frac{\left(\varrho_{+}^{-2}(r)+u_{+}^{\prime 2}(r)\right)^{1 / 2}}{\left(\varrho^{-2}(x)+u_{+}^{\prime 2}(r)\right)^{1 / 2}}\right)-n H .
\end{aligned}
$$

Moreover, suppose that

$$
\frac{\partial_{r} \varrho(x)}{\varrho(x)} \geq \frac{\varrho_{+}^{\prime}(r(x))}{\varrho_{+}(r(x))}
$$

for some positive and increasing $C^{1}$-function $\varrho_{+}:[0, \infty) \rightarrow(0, \infty)$ such that $\varrho_{+}(0)=\varrho(o)$. By our choice of $u_{+}$,

$$
u_{+}^{\prime}(r)=-\frac{\int_{0}^{r} n \tilde{H}(s) \varrho_{+}(s) \xi_{+}^{n-1}(s) \mathrm{d} s}{\varrho_{+}(r) \sqrt{\varrho_{+}^{2}(r) \xi_{+}^{2(n-1)}(r)-\left(\int_{0}^{r} n \widetilde{H}(s) \varrho_{+}(s) \xi_{+}^{n-1}(s) \mathrm{d} s\right)^{2}}},
$$

and therefore

$$
-n \tilde{H}=\left(\frac{u_{+}^{\prime}(r)}{\left(\varrho_{+}^{-2}(r)+u_{+}^{\prime 2}(r)\right)^{1 / 2}}\right)^{\prime}+\frac{u_{+}^{\prime}(r)}{\left(\varrho_{+}^{-2}(r)+u_{+}^{\prime 2}(r)\right)^{1 / 2}}\left(\frac{\varrho_{+}^{\prime}(r)}{\varrho_{+}(r)}+(n-1) \frac{\xi_{+}^{\prime}(r)}{\xi_{+}(r)}\right) .
$$

Hence we obtain

$$
\begin{aligned}
\mathcal{Q}\left[u_{+}\right]= & \frac{\left(\varrho_{+}^{-2}(r)+u_{+}^{\prime 2}(r)\right)^{1 / 2}}{\left(\varrho^{-2}(x)+u_{+}^{\prime 2}(r)\right)^{1 / 2}}\left[\frac{u_{+}^{\prime}(r)}{\left(\varrho_{+}^{-2}(r)+u_{+}^{\prime 2}(r)\right)^{1 / 2}}\left(\Delta r+\frac{1}{\varrho}\left\langle\nabla \varrho, \partial_{r}\right\rangle\right)\right. \\
& \left.+\partial_{r}\left(\frac{u_{+}^{\prime}(r)}{\left(\varrho_{+}^{-2}(r)+u_{+}^{\prime 2}(r)\right)^{1 / 2}}\right)\right]+\frac{u_{+}^{\prime}(r)}{\left(\varrho_{+}^{-2}(r)+u_{+}^{\prime 2}(r)\right)^{1 / 2}} \partial_{r}\left(\frac{\left(\varrho_{+}^{-2}(r)+u_{+}^{\prime 2}(r)\right)^{1 / 2}}{\left(\varrho^{-2}(x)+u_{+}^{\prime 2}(r)\right)^{1 / 2}}\right)-n H \\
\leq & -\frac{\left(\varrho_{+}^{-2}(r)+u_{+}^{\prime 2}(r)\right)^{1 / 2}}{\left(\varrho^{-2}(x)+u_{+}^{\prime 2}(r)\right)^{1 / 2}} n \widetilde{H}+\frac{u_{+}^{\prime}(r)}{\left(\varrho_{+}^{-2}(r)+u_{+}^{\prime 2}(r)\right)^{1 / 2}} \partial_{r}\left(\frac{\left(\varrho_{+}^{-2}(r)+u_{+}^{\prime 2}(r)\right)^{1 / 2}}{\left(\varrho^{-2}(x)+u_{+}^{\prime 2}(r)\right)^{1 / 2}}\right)-n H .
\end{aligned}
$$

In order to prove that $u_{+}$is indeed an upper barrier we next check that

$$
\partial_{r}\left(\frac{\left(\varrho_{+}^{-2}(r)+u_{+}^{\prime 2}(r)\right)^{1 / 2}}{\left(\varrho^{-2}(x)+u_{+}^{\prime 2}(r)\right)^{1 / 2}}\right) \geq 0 .
$$

Note that $u_{+}^{\prime} \leq 0$. We observe that

$$
\frac{\partial}{\partial r}\left(\sqrt{\frac{\varrho_{+}(r)^{-2}+\left(u_{+}^{\prime}(r)\right)^{2}}{\varrho(x)^{-2}+\left(u_{+}^{\prime}(r)\right)^{2}}}\right) \geq 0
$$

if and only if

$$
\left(\varrho_{+}^{-2}+\left(u_{+}^{\prime}\right)^{2}\right)\left(\frac{\partial_{r} \varrho}{\varrho^{3}}-u_{+}^{\prime} u_{+}^{\prime \prime}\right) \geq\left(\varrho^{-2}+\left(u_{+}^{\prime}\right)^{2}\right)\left(\frac{\varrho_{+}^{\prime}}{\varrho_{+}^{3}}-u_{+}^{\prime} u_{+}^{\prime \prime}\right) .
$$

But now integrating (4.8) we get

$$
\log \varrho(x) \geq \log \varrho_{+}(r(x))
$$

which implies

$$
\frac{1}{\varrho(x)} \leq \frac{1}{\varrho_{+}(r(x))}
$$


and furthermore assuming

$$
\frac{\partial_{r} \varrho(x)}{\varrho(x)^{3}} \geq \frac{\varrho_{+}^{\prime}(r(x))}{\varrho_{+}(r(x))^{3}}
$$

we see that (4.10) holds.

Therefore we are left to show that

$$
-n H \leq \sqrt{\frac{\varrho_{+}^{-2}(r)+u_{+}^{\prime 2}(r)}{\varrho^{-2}(x)+u_{+}^{\prime 2}(r)}} n \tilde{H} .
$$

The conditions (4.4) and (4.5) in our mind, we choose $\widetilde{H}$ as

$$
n \tilde{H}(r)=(1-\varepsilon)\left(\frac{\varrho_{+}^{\prime}(r)}{\varrho_{+}(r)}+(n-1) \frac{\xi_{+}^{\prime}(r)}{\xi_{+}(r)}\right)
$$

with some $\varepsilon \in(0,1)$. Note that then

$$
\int_{0}^{r} n \widetilde{H}(s) \varrho_{+}(s) \xi_{+}^{n-1}(s) \mathrm{d} s=(1-\varepsilon) \varrho_{+}(r) \xi_{+}^{n-1}(r)
$$

and we see that with this choice the denominator in the definition of $u_{+}$stays bounded from 0 . Moreover, we have

$$
u_{+}^{\prime}(r)=-\frac{1-\varepsilon}{\varrho_{+}(r) \sqrt{2 \varepsilon-\varepsilon^{2}}}
$$

and therefore $u_{+}$is well defined, positive and decreasing function if

$$
\int_{1}^{\infty} \frac{1}{\varrho_{+}(r)} \mathrm{d} r<\infty
$$

Now we can compute

$$
\begin{aligned}
\frac{\varrho_{+}^{-2}(r)+u_{+}^{\prime 2}(r)}{\varrho^{-2}(x)+u_{+}^{\prime 2}(r)} & =\frac{\varrho_{+}^{-2}(r)+\left((1-\varepsilon) /\left(\varrho_{+}(r) \sqrt{2 \varepsilon-\varepsilon^{2}}\right)\right)^{2}}{\varrho^{-2}(x)+\left((1-\varepsilon) /\left(\varrho_{+}(r) \sqrt{2 \varepsilon-\varepsilon^{2}}\right)\right)^{2}} \\
& =\frac{\varrho_{+}^{-2}(r)\left(1+(1-\varepsilon)^{2} /\left(2 \varepsilon-\varepsilon^{2}\right)\right)}{\varrho^{-2}(x)+\varrho_{+}^{-2}(r)\left(1-\varepsilon^{2}\right) /\left(2 \varepsilon-\varepsilon^{2}\right)},
\end{aligned}
$$

and for example, taking $\varepsilon=1-\sqrt{2} / 2$ we have

$$
\frac{\varrho_{+}^{-2}(r)+u_{+}^{\prime 2}(r)}{\varrho^{-2}(x)+u_{+}^{\prime 2}(r)}=\frac{2 \varrho_{+}^{-2}(r)}{\varrho^{-2}(x)+\varrho_{+}^{-2}(r)} .
$$

For the prescribed mean curvature we obtain the bound

$$
-n H(x) \leq(1-\varepsilon) \sqrt{\frac{\varrho_{+}^{-2}(r)\left(1+(1-\varepsilon)^{2} /\left(2 \varepsilon-\varepsilon^{2}\right)\right)}{\varrho^{-2}(x)+\varrho_{+}^{-2}(r)\left(1-\varepsilon^{2}\right) /\left(2 \varepsilon-\varepsilon^{2}\right)}}\left(\frac{\varrho_{+}^{\prime}(r)}{\varrho_{+}(r)}+(n-1) \frac{\xi_{+}^{\prime}(r)}{\xi_{+}(r)}\right)
$$

which implies that $Q\left[u_{+}\right] \leq 0$. Similarly, $Q\left[-u_{+}\right] \geq 0$ if

$$
n H(x) \leq(1-\varepsilon) \sqrt{\frac{\varrho_{+}^{-2}(r)\left(1+(1-\varepsilon)^{2} /\left(2 \varepsilon-\varepsilon^{2}\right)\right)}{\varrho^{-2}(x)+\varrho_{+}^{-2}(r)\left(1-\varepsilon^{2}\right) /\left(2 \varepsilon-\varepsilon^{2}\right)}}\left(\frac{\varrho_{+}^{\prime}(r)}{\varrho_{+}(r)}+(n-1) \frac{\xi_{+}^{\prime}(r)}{\xi_{+}(r)}\right) .
$$


All together, we have obtained the following.

Lemma 4.1 Let $M$ be a complete Riemannian manifold with a pole o and consider the warped product manifold $M \times_{\varrho} \mathbb{R}$, where $\varrho$ satisfies

$$
\frac{\partial_{r} \varrho(x)}{\varrho(x)} \geq \frac{\varrho_{+}^{\prime}(r(x))}{\varrho_{+}(r(x))}, \quad \frac{\partial_{r} \varrho(x)}{\varrho(x)^{3}} \geq \frac{\varrho_{+}^{\prime}(r(x))}{\varrho_{+}(r(x))^{3}}
$$

for some positive and increasing $C^{1}$-function $\varrho_{+}:[0, \infty) \rightarrow(0, \infty)$ such that

$$
\varrho_{+}(0)=\varrho(o) \text { and } \int_{1}^{\infty} \varrho_{+}(s)^{-1} \mathrm{~d} s<\infty .
$$

Furthermore, assume that the radial sectional curvatures of $M$ are bounded from above by

$$
K_{M}\left(P_{x}\right) \leq-\frac{\xi_{+}^{\prime \prime}(r(x))}{\xi_{+}(r(x))}
$$

and that the prescribed mean curvature function satisfies

$$
\begin{aligned}
& n|H(x)| \\
& \quad \leq(1-\varepsilon) \sqrt{\frac{\varrho_{+}^{-2}(r(x))\left(1+(1-\varepsilon)^{2} /\left(2 \varepsilon-\varepsilon^{2}\right)\right)}{\varrho^{-2}(x)+\varrho_{+}^{-2}(r(x))\left(1-\varepsilon^{2}\right) /\left(2 \varepsilon-\varepsilon^{2}\right)}}\left(\frac{\varrho_{+}^{\prime}((r))}{\varrho_{+}(r(x))}+(n-1) \frac{\xi_{+}^{\prime}(r(x))}{\xi_{+}(r(x))}\right)
\end{aligned}
$$

for some $\varepsilon \in(0,1)$. Then the function $u_{+}$defined by (4.6) and (4.11) satisfies $Q\left[u_{+}\right] \leq 0$ and $u_{+} \geq\|\varphi\|_{C^{0}}$ in $M$ with

$$
u_{+}(r) \rightarrow\|\varphi\|_{C^{0}} \text { as } r \rightarrow \infty .
$$

Furthermore $Q\left[-u_{+}\right] \geq 0$ and $-u_{+} \leq-\|\varphi\|_{C^{0}}$ in $M$.

Remark 4.2 In particular, if the sectional curvatures of a Cartan-Hadamard manifold $M$ are bounded from above as

$$
K_{M}\left(P_{x}\right) \leq-a(r(x))^{2}
$$

for some smooth function $a:[0, \infty) \rightarrow[0, \infty)$, the condition (4.15) reads as

$$
\begin{aligned}
& n|H(x)| \\
& \quad \leq(1-\varepsilon) \sqrt{\frac{\varrho_{+}^{-2}(r(x))\left(1+(1-\varepsilon)^{2} /\left(2 \varepsilon-\varepsilon^{2}\right)\right)}{\varrho^{-2}(x)+\varrho_{+}^{-2}(r(x))\left(1-\varepsilon^{2}\right) /\left(2 \varepsilon-\varepsilon^{2}\right)}}\left(\frac{\varrho_{+}^{\prime}((r))}{\varrho_{+}(r(x))}+(n-1) \frac{f_{a}^{\prime}(r(x))}{f_{a}(r(x))}\right),
\end{aligned}
$$

with $f_{a}$ as in (2.3).

In a rotationally symmetric case if $\varrho=\varrho_{+}(r)$ (and (4.12) holds), we see that the bound for the mean curvature is

$$
n|H(x)| \leq(1-\varepsilon)\left(\frac{\varrho_{+}^{\prime}(r(x))}{\varrho_{+}(r(x))}+(n-1) \frac{\xi_{+}^{\prime}((r(x)}{\xi_{+}(r(x))}\right) .
$$




\subsection{Example: hyperbolic space}

We consider the warped model of $\mathbb{H}^{n+1}$ given by $\mathbb{H}^{n} \times \cosh r \mathbb{R}$, where $r$ is a radial coordinate in $\mathbb{H}^{n}$ defined with respect to a fixed reference point $o \in \mathbb{H}^{n}$. Then the hyperbolic metric is expressed as

$$
\cosh ^{2} \mathrm{~d} t^{2}+\mathrm{d} r^{2}+\sinh ^{2} r \mathrm{~d} \vartheta^{2}
$$

where $\mathrm{d} \vartheta^{2}$ stands for the standard metric in $\mathbb{S}^{n-1} \subset T_{o} \mathbb{H}^{n}$. The flow of the Killing field $X=\partial_{t}$ is given by the hyperbolic translations generated by a geodesic $\gamma$ orthogonal to $\mathbb{H}^{n}$ through $o$. Since $\varrho(r)=\cosh r$ and $\xi(r)=\sinh r$ in this case, we obtain

$$
\begin{aligned}
& \lim _{r \rightarrow \infty} \frac{\varrho(r) \xi^{n-1}(r)}{\int_{0}^{r} \varrho(\tau) \xi^{n-1}(\tau) \mathrm{d} \tau}=\lim _{r \rightarrow \infty} \frac{\sinh ^{n} r+(n-1) \cosh ^{2} r \sinh ^{n-2} r}{\cosh r \sinh ^{n-1} r} \\
& \quad=\lim _{r \rightarrow \infty}\left(\frac{\sinh r}{\cosh r}+(n-1) \frac{\cosh r}{\sinh r}\right) \geq n .
\end{aligned}
$$

Therefore a natural bound to the mean curvature function according (4.4) is

$$
|H|<1,
$$

that is, below the mean curvature of horospheres.

We also have for $|H|<1$

$$
\frac{I^{2}(r) \varrho^{-2}(r)}{\varrho^{2}(r) \xi^{2(n-1)}(r)-I^{2}(r)} \leq \frac{\sinh ^{2 n} r \cosh ^{-2}(r)}{\cosh ^{2} r \sinh ^{2(n-1)} r-\sinh ^{2 n} r}=\frac{\sinh ^{2} r}{\cosh ^{2} r} .
$$

Therefore we have

$$
u^{\prime 2}(r) \leq 1
$$

If $|H|=$ cte. $<1$ we have an explicit expression

$$
u^{\prime 2}(r)=\frac{H^{2}}{\cosh ^{2} r-H^{2} \sinh ^{2} r} \frac{\cosh ^{2} r}{\sinh ^{2} r} .
$$

\subsection{Global barrier $V$}

In this subsection we construct a global barrier using an idea of Mastrolia, Monticelli, and Punzo [15]; see also [4]. Recall that $\varrho_{+}:[0, \infty) \rightarrow(0, \infty)$ is an increasing smooth function satisfying $\varrho_{+}(0)=\varrho(o)$ and

$$
\frac{\partial_{r} \varrho(x)}{\varrho(x)} \geq \frac{\varrho_{+}^{\prime}(r(x))}{\varrho_{+}(r(x))}
$$

for all $x \in M$. Then we have an estimate

$$
\Delta_{-\log \varrho} r(x) \geq(n-1) \frac{f_{a}^{\prime}(r(x))}{f_{a}(r(x))}+\frac{\varrho_{+}^{\prime}(r(x))}{\varrho_{+}(r(x))}
$$

for the weighted Laplacian of the distance function $r$. Let $a_{0}$ be a positive function such that

$$
\int_{0}^{\infty}\left(\int_{t}^{\infty} \frac{\mathrm{d} s}{\varrho_{+}^{2}(s) f_{a}^{n-1}(s)}\right) a_{0}(t) f_{a}^{n-1}(t) \mathrm{d} t<\infty .
$$


We define

$$
\begin{aligned}
V(x)= & \left(\int_{r(x)}^{\infty} \frac{\mathrm{d} s}{\varrho_{+}^{2}(s) f_{a}^{n-1}(s)}\right)\left(\int_{0}^{r(x)} a_{0}(t) f_{a}^{n-1}(t) \mathrm{d} t\right) \\
& -\int_{0}^{r(x)}\left(\int_{t}^{\infty} \frac{\mathrm{d} s}{\varrho_{+}^{2}(s) f_{a}^{n-1}(s)}\right) a_{0}(t) f_{a}^{n-1}(t) \mathrm{d} t-D+\|\varphi\|_{\infty},
\end{aligned}
$$

where $D$ is the constant given by (4.23). Denoting $V(r)=V(r(x))$, we observe that

$$
V^{\prime}(r)=-\frac{1}{\varrho_{+}^{2}(r) f_{a}^{n-1}(r)} \int_{0}^{r} a_{0}(t) f_{a}^{n-1}(t) \mathrm{d} t<0
$$

and

$$
V^{\prime \prime}(r)=\frac{1}{\varrho_{+}^{2}(r) f_{a}^{n-1}(r)}\left(\frac{(n-1) f_{a}^{\prime}(r)}{f_{a}(r)}+\frac{2 \varrho_{+}^{\prime}(r)}{\varrho_{+}(r)}\right) \int_{0}^{r} a_{0}(t) f_{a}^{n-1}(t) \mathrm{d} t-\frac{a_{0}(r)}{\varrho_{+}^{2}(r)} .
$$

Since $V^{\prime}(r)<0$, the limit

$$
\begin{aligned}
D= & \lim _{r \rightarrow \infty}\left\{\int_{r}^{\infty} \frac{\mathrm{d} s}{\varrho_{+}^{2}(s) f_{a}^{n-1}(s)} \int_{0}^{r} a_{0}(t) f_{a}^{n-1}(t) \mathrm{d} t\right. \\
& \left.-\int_{0}^{r} \int_{t}^{\infty} \frac{\mathrm{d} s}{\varrho_{+}^{2}(s) f_{a}^{n-1}(s)} a_{0}(t) f_{a}^{n-1}(t) \mathrm{d} t\right\}
\end{aligned}
$$

exists. Furthermore, $D \leq 0$ (see [15, (4.5)]) and finite by (4.21) and therefore $V$ is well defined. Next we write

$$
\begin{aligned}
& \mathcal{Q}[V] \\
& =\frac{\left(\varrho^{-2}+|\nabla V|^{2}\right) \Delta_{-\log \varrho} V-\left(\varrho^{-2}+|\nabla V|^{2}\right)^{3 / 2} n H(x)-\frac{1}{2}\left\langle\nabla\left(\varrho^{-2}+|\nabla V|^{2}\right), \nabla V\right\rangle}{\left(\varrho^{-2}+|\nabla V|^{2}\right)^{3 / 2}}
\end{aligned}
$$

and aim to prove that $\mathcal{Q}[V] \leq 0$. First we estimate the weighted Laplacian of $V$ by using (4.20)

$$
\begin{aligned}
\Delta_{-\log \varrho} V= & V^{\prime \prime}(r)+V^{\prime}(r) \Delta_{-} \log \varrho^{r} \\
\leq & V^{\prime \prime}(r)+\left((n-1) \frac{f_{a}^{\prime}(r)}{f_{a}(r)}+\frac{\varrho_{+}^{\prime}(r)}{\varrho_{+}(r)}\right) V^{\prime}(r) \\
= & \frac{1}{\varrho_{+}^{2}(r) f_{a}^{n-1}(r)}\left(\frac{(n-1) f_{a}^{\prime}(r)}{f_{a}(r)}+\frac{2 \varrho_{+}^{\prime}(r)}{\varrho_{+}(r)}\right) \int_{0}^{r} a_{0}(t) f_{a}^{n-1}(t) \mathrm{d} t \\
& -\frac{a_{0}(r)}{\varrho_{+}^{2}(r)}-\frac{1}{\varrho_{+}^{2}(r) f_{a}^{n-1}(r)}\left(\frac{(n-1) f_{a}^{\prime}(r)}{f_{a}(r)}+\frac{\varrho_{+}^{\prime}(r)}{\varrho_{+}(r)}\right) \int_{0}^{r} a_{0}(t) f_{a}^{n-1}(t) \mathrm{d} t \\
= & -\frac{a_{0}(r)}{\varrho_{+}^{2}(r)}+\frac{\varrho_{+}^{\prime}(r)}{\varrho_{+}^{3}(r) f_{a}^{n-1}(r)} \int_{0}^{r} a_{0}(t) f_{a}^{n-1}(t) \mathrm{d} t \\
= & -\frac{a_{0}(r)}{\varrho_{+}^{2}(r)}-\frac{\varrho_{+}^{\prime}(r)}{\varrho_{+}(r)} V^{\prime}(r),
\end{aligned}
$$


and thus the first term of (4.24) can be estimated as

$$
\left(\varrho^{-2}+|\nabla V|^{2}\right) \Delta_{-\log \varrho} V \leq-\left(\varrho^{-2}+\left(V^{\prime}(r)\right)^{2}\right)\left(\frac{a_{0}(r)}{\varrho_{+}^{2}(r)}+\frac{\varrho_{+}^{\prime}(r)}{\varrho_{+}(r)} V^{\prime}(r)\right) .
$$

Then, for the last term of (4.24) we have

$$
\begin{aligned}
& -\frac{1}{2}\left\langle\nabla\left(\varrho^{-2}+|\nabla V|^{2}\right), \nabla V\right\rangle=-\left(V^{\prime}(r)\right)^{2} V^{\prime \prime}(r)+\frac{\partial_{r} \varrho}{\varrho^{3}} V^{\prime}(r) \\
& =-\left(V^{\prime}(r)\right)^{2}\left(\left(\frac{(n-1) f_{a}^{\prime}(r)}{f_{a}(r)}+\frac{2 \varrho_{+}^{\prime}(r)}{\varrho_{+}(r)}\right) V^{\prime}(r)-\frac{a_{0}(r)}{\varrho_{+}^{2}(r)}\right)+\frac{\partial_{r} \varrho}{\varrho^{3}} V^{\prime}(r) .
\end{aligned}
$$

Hence

$$
\begin{aligned}
\varrho_{+}^{2} & (r)\left(\varrho^{-2}+|\nabla V|^{2}\right) \Delta_{-} \log \varrho V-\frac{1}{2} \varrho_{+}^{2}(r)\left\langle\nabla\left(\varrho^{-2}+|\nabla V|^{2}\right), \nabla V\right\rangle \\
\leq & -\varrho^{-2} a_{0}(r)-\varrho^{-2} \varrho_{+}^{2}(r)\left(\frac{\varrho_{+}^{\prime}(r)}{\varrho_{+}(r)}-\frac{\partial_{r} \varrho}{\varrho}\right) V^{\prime}(r) \\
& -\varrho_{+}^{2}(r)\left(V^{\prime}(r)\right)^{3}\left(\frac{(n-1) f_{a}^{\prime}(r)}{f_{a}(r)}+\frac{\varrho_{+}^{\prime}(r)}{\varrho_{+}(r)}\right) \\
& \leq-\varrho^{-2} a_{0}(r)-\varrho_{+}^{2}(r)\left(V^{\prime}(r)\right)^{3}\left(\frac{(n-1) f_{a}^{\prime}(r)}{f_{a}(r)}+\frac{\varrho_{+}^{\prime}(r)}{\varrho_{+}(r)}\right) .
\end{aligned}
$$

Finally, if the prescribed mean curvature function satisfies

$$
-n H \leq \frac{\varrho^{-2} \varrho_{+}^{-2}(r) a_{0}(r)+\left(-V^{\prime}(r)\right)^{3}\left(\frac{(n-1) f_{a}^{\prime}(r)}{f_{a}(r)}+\frac{\varrho_{+}^{\prime}(r)}{\varrho_{+}(r)}\right)}{\left(\varrho^{-2}+\left(V^{\prime}(r)\right)^{2}\right)^{3 / 2}}
$$

in $M$, we obtain $\mathcal{Q}[V] \leq 0$ as desired. Similarly, we see that $\mathcal{Q}[-V] \geq 0$ if

$$
n H \leq \frac{\varrho^{-2} \varrho_{+}^{-2}(r) a_{0}(r)+\left(-V^{\prime}(r)\right)^{3}\left(\frac{(n-1) f_{a}^{\prime}(r)}{f_{a}(r)}+\frac{\varrho_{+}^{\prime}(r)}{\varrho_{+}(r)}\right)}{\left(\varrho^{-2}+\left(V^{\prime}(r)\right)^{2}\right)^{3 / 2}} .
$$

Hence we have proved the following uniform height estimate.

Lemma 4.3 Let $\varphi: M \rightarrow \mathbb{R}$ be a bounded function and assume that the prescribed mean curvature function $H$ and the function $V$ defined in (4.22) satisfy

$$
n|H| \leq \frac{\varrho^{-2} \varrho_{+}^{-2}(r) a_{0}(r)+\left(-V^{\prime}(r)\right)^{3}\left(\frac{(n-1) f_{a}^{\prime}(r)}{f_{a}(r)}+\frac{\varrho_{+}^{\prime}(r)}{\varrho_{+}(r)}\right)}{\left(\varrho^{-2}+\left(V^{\prime}(r)\right)^{2}\right)^{3 / 2}},
$$

with some positive functions $\varrho_{+}$and $a_{0}$ satifying (4.19) and (4.21), respectively. Then

$$
\begin{aligned}
& \mathcal{Q}[V]=\operatorname{div}_{-\log \varrho} \frac{\nabla V}{\sqrt{\varrho^{-2}+|\nabla V|^{2}}}-n H \leq 0 \text { in } M, \\
& V(x)>\|\varphi\|_{\infty} \text { for all } x \in M,
\end{aligned}
$$


and

$$
\lim _{r(x) \rightarrow \infty} V(x)=\|\varphi\|_{\infty} .
$$

Furthermore, $Q[-V] \geq 0$ in $M$.

Next we discuss possible choices of the functions $\varrho_{+}$and $a_{0}$ and their influence on the bound of $|H|$. Notice that the right hand side of (4.25) can be written as

$$
\frac{\frac{\varrho \varrho_{+}^{-2}(r) a_{0}(r)}{\left(-V^{\prime}(r) \varrho\right)^{3}}+\frac{(n-1) f_{a}^{\prime}(r)}{f_{a}(r)}+\frac{\varrho_{+}^{\prime}(r)}{\varrho_{+}(r)}}{\left(1+\left(-V^{\prime}(r) \varrho\right)^{-2}\right)^{3 / 2}} .
$$

Hence if we can choose the comparison manifold $M_{-a^{2}(r)} \times \varrho_{+} \mathbb{R}$ and $a_{0}$ such that $V^{\prime}(r) \varrho \rightarrow$ $-\infty$ and

$$
\frac{\varrho \varrho_{+}^{-2}(r) a_{0}(r)}{\left(-V^{\prime}(r) \varrho\right)^{3}} \rightarrow 0
$$

as $r \rightarrow \infty$, we obtain

$$
n|H| \leq \frac{(n-1) f_{a}^{\prime}(r)}{f_{a}(r)}+\frac{\varrho_{+}^{\prime}(r)}{\varrho_{+}(r)}
$$

asymptotically as $r \rightarrow \infty$.

Example 4.4 In the hyperbolic case $\mathbb{H}^{n+1}=\mathbb{H}^{n} \times \cosh r \mathbb{R}$ we may take $\varrho_{+}(r)=\varrho=$ cosh. Choosing $a_{0}(r)=\sinh ^{\alpha} r$ for some $\alpha \in(1,2)$ yields to the natural asymptotic bound $|H|<1$ as $r \rightarrow \infty$.

Example 4.5 More generally, if $N=M \times_{\varrho} \mathbb{R}$, where the sectional curvatures of $M$ have a negative upper bound $-k^{2}$ and if the warping function $\varrho$ satisfies (4.19) with $\varrho_{+}(r) \geq c_{1} e^{\alpha r}$ for some $\alpha>0$, then $f_{a}(r) \approx e^{k r}$ and (4.21) holds if

$$
\int_{0}^{\infty} a_{0}(t) e^{-2 \alpha t} \mathrm{~d} t<\infty
$$

Moreover, if $\varrho_{+}(r) \leq c_{2} e^{\beta r}$ for some $0<\beta<2 \alpha$, then by choosing $a_{0}(t)=e^{\kappa t}, \beta<\kappa<$ $2 \alpha$, we get (4.30) asymptotically as $r \rightarrow \infty$.

Example 4.6 If $N=M \times_{\varrho} \mathbb{R}$, where the sectional curvatures of $M$ have a negative upper bound

$$
K\left(P_{x}\right) \leq-\frac{\phi(\phi-1)}{r(x)^{2}}, \quad \phi>1,
$$

and if the warping function $\varrho$ satisfies (4.19) with $\varrho_{+}(r)=c r^{\alpha}, \alpha>1$, then $f_{a}(r) \approx r^{\phi}$ and (4.21) holds if

$$
\int_{0}^{\infty} a_{0}(r) r^{-2 \alpha+1} \mathrm{~d} r<\infty .
$$

Choosing $a_{0}(r)=r^{\kappa}$, for some $\alpha-1<\kappa<2(\alpha-1)$, we get (4.30) asymptotically as $r \rightarrow \infty$. 


\section{Barrier at infinity}

In this section we assume that $M$ is a Cartan-Hadamard manifold of dimension $n \geq 2$, $\partial_{\infty} M$ is the asymptotic boundary of $M$, and $\bar{M}=M \cup \partial_{\infty} M$ the compactification of $M$ in the cone topology. Recall that the asymptotic boundary is defined as the set of all equivalence classes of unit speed geodesic rays in $M$; two such rays $\gamma_{1}$ and $\gamma_{2}$ are equivalent if $\sup _{t \geq 0} d\left(\gamma_{1}(t), \gamma_{2}(t)\right)<\infty$. The equivalence class of $\gamma$ is denoted by $\gamma(\infty)$. For each $x \in M$ and $y \in \bar{M} \backslash\{x\}$ there exists a unique unit speed geodesic $\gamma^{x, y}: \mathbb{R} \rightarrow M$ such that $\gamma_{0}^{x, y}=x$ and $\gamma_{t}^{x, y}=y$ for some $t \in(0, \infty]$. If $v \in T_{x} M \backslash\{0\}, \alpha>0$, and $r>0$, we define a cone

$$
C(v, \alpha)=\left\{y \in \bar{M} \backslash\{x\}: \varangle\left(v, \dot{\gamma}_{0}^{x, y}\right)<\alpha\right\}
$$

and a truncated cone

$$
T(v, \alpha, r)=C(v, \alpha) \backslash \bar{B}(x, r),
$$

where $\varangle\left(v, \dot{\gamma}_{0}^{x, y}\right)$ is the angle between vectors $v$ and $\dot{\gamma}_{0}^{x, y}$ in $T_{x} M$. All cones and open balls in $M$ form a basis for the cone topology on $\bar{M}$.

Throughout this section, we assume that the sectional curvatures of $M$ are bounded from below and above by

$$
-(b \circ r)^{2}(x) \leq K\left(P_{x}\right) \leq-(a \circ r)^{2}(x)
$$

for all $x \in M$, where $r(x)=d(o, x)$ is the distance to a fixed point $o \in M$ and $P_{x}$ is any 2-dimensional subspace of $T_{x} M$. The functions $a, b:[0, \infty) \rightarrow[0, \infty)$ are assumed to be smooth such that $a(t)=0$ and $b(t)$ is constant for $t \in\left[0, T_{0}\right]$ for some $T_{0}>0$, and that assumptions (A1)-(A7) hold. These curvature bounds are needed to control the first two derivatives of "barrier" functions that we will construct in the next subsection. We assume that function $b$ in (5.1) is monotonic and that there exist positive constants $T_{1} \geq T_{0}, C_{1}, C_{2}, C_{3}$, and $Q \in(0,1)$ such that

$$
a(t) \begin{cases}=C_{1} t^{-1} & \text { if } b \text { is decreasing } \\ \geq C_{1} t^{-1} & \text { if } b \text { is increasing }\end{cases}
$$

for all $t \geq T_{1}$ and

$$
\begin{aligned}
a(t) & \leq C_{2}, \\
b(t+1) & \leq C_{2} b(t), \\
b(t / 2) & \leq C_{2} b(t), \\
b(t) & \geq C_{3}(1+t)^{-Q}
\end{aligned}
$$

for all $t \geq 0$. In addition, we assume that

$$
\lim _{t \rightarrow \infty} \frac{b^{\prime}(t)}{b(t)^{2}}=0
$$

and that there exists a constant $C_{4}>0$ such that

$$
\lim _{t \rightarrow \infty} \frac{t^{1+C_{4}} b(t)}{f_{a}^{\prime}(t)}=0 ;
$$

see (2.3) for the definition of $f_{a}$.

We recall from [13] the following two examples of functions $a$ and $b$. 
Example 5.1 Let $C_{1}=\sqrt{\phi(\phi-1)}$, where $\phi>1$ is a constant. For $t \geq R_{0}$ let

$$
a(t)=\frac{C_{1}}{t}
$$

and

$$
b(t)=t^{\phi-2-\varepsilon / 2},
$$

where $0<\varepsilon<2 \phi-2$, and extend them to smooth functions $a:[0, \infty) \rightarrow(0, \infty)$ and $b:[0, \infty) \rightarrow(0, \infty)$ such that they are constants in some neighborhood of $0, b$ is monotonic and $b \geq a$. Then $a$ and $b$ satisfy (A1)-(A7) with constants $T_{1}=R_{0}, C_{1}$, some $C_{2}>0$, some $C_{3}>0, Q=\max \{1 / 2,-\phi+2+\varepsilon / 2\}$, and any $C_{4} \in(0, \varepsilon / 2)$. It is easy to verify that then

$$
f_{a}(t)=c_{1} t^{\phi}+c_{2} t^{1-\phi}
$$

for all $t \geq R_{0}$, where

$$
c_{1}=R_{0}^{-\phi} \frac{f_{a}\left(R_{0}\right)(\phi-1)+R_{0} f_{a}^{\prime}\left(R_{0}\right)}{2 \phi-1}>0,
$$

and

$$
c_{2}=R_{0}^{\phi-1} \frac{f_{a}\left(R_{0}\right) \phi-R_{0} f_{a}^{\prime}\left(R_{0}\right)}{2 \phi-1} .
$$

We then have

$$
\lim _{t \rightarrow \infty} \frac{t f_{a}^{\prime}(t)}{f_{a}(t)}=\phi
$$

and, for all $C_{4} \in(0, \varepsilon / 2)$

$$
\lim _{t \rightarrow \infty} \frac{t^{1+C_{4}} b(t)}{f_{a}^{\prime}(t)}=0 .
$$

It follows that $a$ and $b$ satisfy (A1)-(A7) with constants $T_{1}=R_{0}, C_{1}$, some $C_{2}>0$, some $C_{3}>0, Q=\max \{1 / 2,-\phi+2+\varepsilon / 2\}$, and any $C_{4} \in(0, \varepsilon / 2)$.

Example 5.2 Let $k>0$ and $\varepsilon>0$ be constants and define $a(t)=k$ for all $t \geq 0$. Define

$$
b(t)=t^{-1-\varepsilon / 2} e^{k t}
$$

for $t \geq R_{0}=r_{0}+1$, where $r_{0}>0$ is so large that $t \mapsto t^{-1-\varepsilon / 2} e^{k t}$ is increasing and greater than $k$ for all $t \geq r_{0}$. Extend $b$ to an increasing smooth function $b:[0, \infty) \rightarrow[k, \infty)$ that is constant in some neighborhood of 0 . We can choose $C_{1}>0$ in (A1) as large as we wish. Then $a$ and $b$ satisfy (A1)-(A7) with constants $C_{1}, T_{1}=C_{1} / k$, some $C_{2}>0$, some $C_{3}>0$, $Q=1 / 2$, and any $C_{4} \in(0, \varepsilon / 2)$.

\subsection{Construction of a barrier}

Following [13], we construct a barrier function for each boundary point $x_{0} \in \partial_{\infty} M$. Towards this end let $v_{0}=\dot{\gamma}_{0}^{o, x_{0}}$ be the initial (unit) vector of the geodesic ray $\gamma^{o, x_{0}}$ from a fixed point $o \in M$ and define a function $h: \partial_{\infty} M \rightarrow \mathbb{R}$,

$$
h(x)=\min \left(1, L \varangle\left(v_{0}, \dot{\gamma}_{0}^{o, x}\right)\right),
$$


where $L \in(8 / \pi, \infty)$ is a constant. Then we define a crude extension $\tilde{h} \in C(\bar{M})$, with $\tilde{h} \mid \partial_{\infty} M=h$, by setting

$$
\tilde{h}(x)=\min \left(1, \max \left(2-2 r(x), L \varangle\left(v_{0}, \dot{\gamma}_{0}^{o, x}\right)\right)\right) .
$$

Finally, we smooth out $\tilde{h}$ to get an extension $h \in C^{\infty}(M) \cap C(\bar{M})$ with controlled first and second order derivatives. For that purpose, we fix $\chi \in C^{\infty}(\mathbb{R})$ such that $0 \leq \chi \leq 1$, $\operatorname{supp} \chi \subset[-2,2]$, and $\chi \mid[-1,1] \equiv 1$. Then for any function $\varphi \in C(M)$ we define functions $F_{\varphi}: M \times M \rightarrow \mathbb{R}, \mathcal{R}(\varphi): M \rightarrow M$, and $\mathcal{P}(\varphi): M \rightarrow \mathbb{R}$ by

$$
\begin{aligned}
F_{\varphi}(x, y) & =\chi(b(r(y)) d(x, y)) \varphi(y), \\
\mathcal{R}(\varphi)(x) & =\int_{M} F_{\varphi}(x, y) \mathrm{d} m(y), \text { and } \\
\mathcal{P}(\varphi) & =\frac{\mathcal{R}(\varphi)}{\mathcal{R}(1)},
\end{aligned}
$$

where

$$
\mathcal{R}(1)(x)=\int_{M} \chi(b(r(y)) d(x, y)) \mathrm{d} m(y)>0 .
$$

Thus $\mathcal{P}(\varphi)$ is an integral average of $\varphi$ with respect to $\chi$ similar to that in $[1$, p. 436] except that here the function $b$ is taken into account explicitly. If $\varphi \in C(\bar{M})$, we extend $\mathcal{P}(\varphi): M \rightarrow \mathbb{R}$ to a function $\bar{M} \rightarrow \mathbb{R}$ by setting $\mathcal{P}(\varphi)(x)=\varphi(x)$ whenever $x \in M(\infty)$. Then the extended function $\mathcal{P}(\varphi)$ is $C^{\infty}$-smooth in $M$ and continuous in $\bar{M}$; see [13, Lemma 3.13]. In particular, applying $\mathcal{P}$ to the function $\tilde{h}$ yields an appropriate smooth extension

$$
h:=\mathcal{P}(\tilde{h})
$$

of the original function $h \in C\left(\partial_{\infty} M\right)$ that was defined in (5.2).

We denote

$$
\Omega=C\left(v_{0}, 1 / L\right) \cap M \text { and } \ell \Omega=C\left(v_{0}, \ell / L\right) \cap M
$$

for $\ell>0$. We collect together all these constants and functions and denote

$$
C=\left(a, b, T_{1}, C_{1}, C_{2}, C_{3}, C_{4}, Q, n, L\right) .
$$

Furthermore, we denote by $\left\|\operatorname{Hess}_{x} u\right\|$ the norm of the Hessian of a smooth function $u$ at $x$, that is

$$
\left\|\operatorname{Hess}_{x} u\right\|=\sup _{\substack{X \in T_{X} M \\|X| \leq 1}}|\operatorname{Hess} u(X, X)| .
$$

The following lemma gives the desired estimates for derivatives of $h$. We refer to [13] for the proofs of these estimates; see also [6].

Lemma 5.3 [13, Lemma 3.16] There exist constants $R_{1}=R_{1}(C)$ and $c_{1}=c_{1}(C)$ such that the extended function $h \in C^{\infty}(M) \cap C(\bar{M})$ in (5.4) satisfies

$$
\begin{aligned}
|\nabla h(x)| & \leq c_{1} \frac{1}{\left(f_{a} \circ r\right)(x)}, \\
\left\|\operatorname{Hess}_{x} h\right\| & \leq c_{1} \frac{(b \circ r)(x)}{\left(f_{a} \circ r\right)(x)},
\end{aligned}
$$


for all $x \in 3 \Omega \backslash B\left(o, R_{1}\right)$. In addition,

$$
h(x)=1
$$

for every $x \in M \backslash\left(2 \Omega \cup B\left(o, R_{1}\right)\right)$.

Let $A>0$ be a fixed constant, and $R_{3}>0$ and $\delta>0$ constants that will be determined later, and $h$ the function defined in (5.4). We will show that a function

$$
\psi=A\left(R_{3}^{\delta} r^{-\delta}+h\right)
$$

is a supersolution

$$
\begin{aligned}
\mathcal{Q}[\psi] & =\operatorname{div}_{-} \log \varrho \frac{\nabla \psi}{\sqrt{\varrho^{-2}+|\nabla \psi|^{2}}}-n H \\
& =\operatorname{div} \frac{\nabla \psi}{W}+\left\langle\nabla \log \varrho, \frac{\nabla \psi}{W}\right\rangle-n H<0
\end{aligned}
$$

in the $3 \Omega \backslash \bar{B}\left(o, R_{3}\right)$. In the proof we shall use the following estimates obtained in [13]:

Lemma 5.4 [13, Lemma 3.17] There exist constants $R_{2}=R_{2}(C)$ and $c_{2}=c_{2}(C)$ with the following property. If $\delta \in(0,1)$, then

$$
\begin{aligned}
|\nabla h| & \leq c_{2} /\left(f_{a} \circ r\right), \\
\| \text { Hess } h \| & \leq c_{2} r^{-C_{4}-1}\left(f_{a}^{\prime} \circ r\right) /\left(f_{a} \circ r\right), \\
|\nabla\langle\nabla h, \nabla h\rangle| & \leq c_{2} r^{-C_{4}-2}\left(f_{a}^{\prime} \circ r\right) /\left(f_{a} \circ r\right), \\
\left|\nabla\left\langle\nabla h, \nabla\left(r^{-\delta}\right)\right\rangle\right| & \leq c_{2} r^{-C_{4}-2}\left(f_{a}^{\prime} \circ r\right) /\left(f_{a} \circ r\right), \\
\nabla\left\langle\nabla\left(r^{-\delta}\right), \nabla\left(r^{-\delta}\right)\right\rangle & =-2 \delta^{2}(\delta+1) r^{-2 \delta-3} \nabla r
\end{aligned}
$$

in the set $3 \Omega \backslash B\left(o, R_{2}\right)$.

Let us denote

$$
\phi=\frac{1+\sqrt{1+4 C_{1}^{2}}}{2}>1, \text { and } \delta_{1}=\min \left\{C_{4} / 2, \frac{-1+(n-1) \phi}{1+(n-1) \phi}\right\} \in(0,1),
$$

where $C_{1}$ and $C_{4}$ are constants defined in (A1) and (A7), respectively.

Lemma 5.5 Assume that the prescribed mean curvature function $H$ satisfies

$$
\sup _{r(x)=t} n|H(x)|<\frac{C_{0} t^{-\delta_{1}-1}}{\sqrt{\varrho^{-2}(t)+\left(C_{0} t^{-\delta-1}\right)^{2}}}\left((n-1) \frac{f_{a}^{\prime}(t)}{f_{a}(t)}+\frac{\partial_{r} \varrho}{\varrho}-\frac{1}{t}\right)
$$

for some positive constants $C_{0}>1$ and $\delta<\min \left\{\delta_{1}, \phi-1\right\}$, and that the warping function @ satisfies

$$
\max \left(0,-\frac{r \partial_{r} \varrho}{\varrho}\right)=o\left(\frac{r f_{a}^{\prime}(r)}{f_{a}(r)}\right)
$$

and

$$
|\nabla \varrho|=o\left(\frac{f_{a}(r)}{r^{\delta+1}}\left|\partial_{r} \varrho\right|\right)
$$

as $r \rightarrow \infty$. Then there exists a constant $R_{3}=R_{3}\left(C, C_{0}, \delta\right) \geq R_{2}$ such that the function $\psi$ defined in (5.6) satisfies $Q[\psi]<0$ in the set $3 \Omega \backslash \bar{B}\left(o, R_{3}\right)$. 
Proof In the proof we will denote by $c$ those positive constants whose actual value is irrelevant and may vary even within a line. Furthermore, the estimates will be done in $3 \Omega \backslash \bar{B}\left(o, R_{3}\right)$, with $R_{3}$ large enough. Note that

$$
\begin{aligned}
& \mathcal{Q}[\psi]=\frac{\Delta_{-\log \varrho} \psi}{\sqrt{\varrho^{-2}+|\nabla \psi|^{2}}}-\frac{1}{2} \frac{\left\langle\nabla\left(\varrho^{-2}+|\nabla \psi|^{2}\right), \nabla \psi\right\rangle}{\left(\varrho^{-2}+|\nabla \psi|^{2}\right)^{3 / 2}}-n H \\
& =\frac{\left(\varrho^{-2}+|\nabla \psi|^{2}\right) \Delta_{-\log \varrho} \psi-\frac{1}{2}\left\langle\nabla\left(\varrho^{-2}+|\nabla \psi|^{2}\right), \nabla \psi\right\rangle-\left(\varrho^{-2}+|\nabla \psi|^{2}\right)^{3 / 2} n H}{\left(\varrho^{-2}+|\nabla \psi|^{2}\right)^{3 / 2}}
\end{aligned}
$$

and hence we only need to find $R_{3}=R_{3}\left(C, C_{0}, \delta\right) \geq R_{2}$ so that

$$
\begin{aligned}
& \left(\varrho^{-2}+|\nabla \psi|^{2}\right)^{3 / 2} \mathcal{Q}[\psi] \\
& =\left(\varrho^{-2}+|\nabla \psi|^{2}\right) \Delta_{-\log \varrho} \psi-\frac{1}{2}\left\langle\nabla\left(\varrho^{-2}+|\nabla \psi|^{2}\right), \nabla \psi\right\rangle-\left(\varrho^{-2}+|\nabla \psi|^{2}\right)^{3 / 2} n H<0
\end{aligned}
$$

holds in the set $3 \Omega \backslash \bar{B}\left(o, R_{3}\right)$.

The function $\psi$ is $C^{\infty}$-smooth and, in $M \backslash\{o\}$, we have

$$
\nabla \psi=A\left(-R_{3}^{\delta} \delta r^{-\delta-1} \nabla r+\nabla h\right) .
$$

By Lemma 5.3, $|\nabla h| \leq c_{1} / f_{a}(r) \leq \delta r^{-\delta-1}$ when $r$ is large enough and $0<\delta<\min \left\{\delta_{1}, \phi-\right.$ $1\}$; see $[13,(3.30)]$. Hence, for any fixed $\varepsilon>0$, we have

$$
\begin{aligned}
|\nabla \psi|^{2} & =\left(A R_{3}^{\delta} \delta\right)^{2} r^{-2 \delta-2}+A^{2}|\nabla h|^{2}-2 A^{2} R_{3}^{\delta} \delta r^{-\delta-1}\langle\nabla r, \nabla h\rangle \\
& \leq A^{2} \delta^{2}\left(R_{3}^{2 \delta}+2 R_{3}^{\delta}+1\right) r^{-2 \delta-2} \\
& \leq(1+\varepsilon)\left(A R_{3}^{\delta} \delta\right)^{2} r^{-2 \delta-2}
\end{aligned}
$$

and

$$
|\nabla \psi|^{2} \geq A^{2} \delta^{2}\left(R_{3}^{2 \delta}-2 R_{3}^{\delta}\right) r^{-2 \delta-2} \geq(1-\varepsilon)\left(A R_{3}^{\delta} \delta\right)^{2} r^{-2 \delta-2}
$$

in $3 \Omega \backslash \bar{B}\left(o, R_{3}\right)$ for $R_{3}$ large enough.

Next we fix $\varepsilon>0$ so that

$$
\varepsilon<1-\frac{\delta+1}{(n-1)(1-\delta) \phi},
$$

which is possible since $\delta<\delta_{1}$. To simplify the notation below, we denote $\tilde{\varepsilon}=\varepsilon \operatorname{sgn}\left(\partial_{r} \varrho\right)$. In order to estimate the first term in the right-hand side of (5.10), we first observe that

$$
-(n-1) \frac{r f_{a}^{\prime}(r)}{f_{a}(r)}-\frac{r \partial_{r} \varrho}{\varrho}+\frac{\delta+1}{1-\varepsilon}<0
$$

for $r \geq R_{3}$ by (5.8) and (5.11); see [13, (3.25)]. Then we can estimate the weighted Laplacian of $\psi$ as

$$
\begin{aligned}
\Delta_{-\log \varrho} \psi & =A R_{3}^{\delta} \Delta_{-\log \varrho} r^{-\delta}+A \Delta_{-\log \varrho} h \\
& =A R_{3}^{\delta}\left(\Delta r^{-\delta}+\frac{1}{\varrho}\left\langle\nabla \varrho, \nabla\left(r^{-\delta}\right)\right\rangle\right)+A\left(\Delta h+\frac{1}{\varrho}\langle\nabla \varrho, \nabla h\rangle\right) \\
& =A R_{3}^{\delta}\left(-\delta r^{-\delta-1} \Delta r-\delta r^{-\delta-1} \frac{1}{\varrho}\langle\nabla \varrho, \nabla r\rangle+\delta(\delta+1) r^{-\delta-2}\right)
\end{aligned}
$$




$$
\begin{aligned}
& +A\left(\Delta h+\frac{1}{\varrho}\langle\nabla \varrho, \nabla h\rangle\right) \\
\leq & A R_{3}^{\delta} \delta\left(-(n-1) \frac{r f_{a}^{\prime}(r)}{f_{a}(r)}-\frac{r \partial_{r} \varrho}{\varrho}+\delta+1\right) r^{-\delta-2} \\
& +A\left(n c_{2} r^{-C_{4}-1} \frac{f_{a}^{\prime}(r)}{f_{a}(r)}+\frac{c_{2}|\nabla \varrho|}{\varrho f_{a}(r)}\right) \\
\leq & A R_{3}^{\delta} \delta\left(\frac{-(1-\varepsilon)(n-1) r f_{a}^{\prime}(r)}{f_{a}(r)}-\frac{(1-\tilde{\varepsilon}) r \partial_{r} \varrho}{\varrho}+\delta+1\right) r^{-\delta-2}<0
\end{aligned}
$$

for $r \geq R_{3}$. In the last step we used (5.8), (5.9), and the fact that $C_{4}>\delta$. Hence

$$
\begin{aligned}
& \left(\varrho^{-2}+|\nabla \psi|^{2}\right) \Delta_{-} \log \varrho \psi \\
& \leq-\left(\varrho^{-2}+(1-\varepsilon)\left(A R_{3}^{\delta} \delta\right)^{2} r^{-2 \delta-2}\right) A R_{3}^{\delta} \delta\left(\frac{(1-\varepsilon)(n-1) r f_{a}^{\prime}(r)}{f_{a}(r)}\right. \\
& \left.\quad+\frac{(1-\tilde{\varepsilon}) r \partial_{r} \varrho}{\varrho}-1-\delta\right) r^{-\delta-2} .
\end{aligned}
$$

To estimate the second term of (5.10) we split it into two parts as

$$
-\frac{1}{2}\left\langle\nabla\left(\varrho^{-2}+|\nabla \psi|^{2}\right), \nabla \psi\right\rangle=-\frac{1}{2}\left\langle\nabla\left(\varrho^{-2}\right), \nabla \psi\right\rangle-\frac{1}{2}\left\langle\nabla|\nabla \psi|^{2}, \nabla \psi\right\rangle .
$$

For the first term, by (5.9) and Lemma 5.4, we have

$$
\begin{aligned}
-\frac{1}{2}\left\langle\nabla\left(\varrho^{-2}\right), \nabla \psi\right\rangle & =\left\langle\frac{\nabla \varrho}{\varrho^{3}}, \nabla \psi\right\rangle=\left\langle\frac{\nabla \varrho}{\varrho^{3}},-A R_{3}^{\delta} \delta r^{-\delta-1} \nabla r\right\rangle+\left\langle\frac{\nabla \varrho}{\varrho^{3}}, A \nabla h\right\rangle \\
& \leq-A R_{3}^{\delta} \delta r^{-\delta-1} \frac{\partial_{r} \varrho}{\varrho^{3}}+c_{2} A \frac{|\nabla \varrho|}{\varrho^{3} f_{a}(r)} \\
& \leq-(1-\tilde{\varepsilon}) A R_{3}^{\delta} \delta r^{-\delta-1} \frac{\partial_{r} \varrho}{\varrho^{3}} .
\end{aligned}
$$

To estimate the second term we note that

$$
\begin{aligned}
\nabla|\nabla \psi|^{2} & =A^{2} \nabla\left\langle R_{3}^{\delta} \nabla\left(r^{-\delta}\right)+\nabla h, R_{3}^{\delta} \nabla\left(r^{-\delta}\right)+\nabla h\right\rangle \\
& =\left(A R_{3}^{\delta}\right)^{2} \nabla\left\langle\nabla\left(r^{-\delta}\right), \nabla\left(r^{-\delta}\right)\right\rangle+2 A^{2} R_{3}^{\delta} \nabla\left\langle\nabla\left(r^{-\delta}\right), \nabla h\right\rangle+A^{2} \nabla\langle\nabla h, \nabla h\rangle
\end{aligned}
$$

and hence, by a straightforward computation using the estimates of Lemma 5.4, we get

$$
\begin{aligned}
-\frac{1}{2}\left\langle\nabla|\nabla \psi|^{2}, \nabla \psi\right\rangle= & -\frac{1}{2}\left(A R_{3}^{\delta}\right)^{2}\left\langle\nabla\left\langle\nabla\left(r^{-\delta}\right), \nabla\left(r^{-\delta}\right)\right\rangle, \nabla \psi\right\rangle \\
& -A^{2} R_{3}^{\delta}\left\langle\nabla\left\langle\nabla\left(r^{-\delta}\right), \nabla h\right\rangle, \nabla \psi\right\rangle-\frac{1}{2} A^{2}\langle\nabla\langle\nabla h, \nabla h\rangle, \nabla \psi\rangle \\
\leq & \left(A R_{3}^{\delta} \delta\right)^{2}(\delta+1) r^{-2 \delta-3}\langle\nabla r, \nabla \psi\rangle+A^{2} R_{3}^{\delta} c_{2} r^{-C_{4}-2} \frac{f_{a}^{\prime}(r)}{f_{a}(r)}|\nabla \psi| \\
& +\frac{1}{2} A^{2} c_{2} r^{-C_{4}-2} \frac{f_{a}^{\prime}(r)}{f_{a}(r)}|\nabla \psi| \\
\leq & \left(A R_{3}^{\delta} \delta\right)^{2}(\delta+1) r^{-2 \delta-3}\left\langle\nabla r,-A R_{3}^{\delta} \delta r^{-\delta-1} \nabla r+A \nabla h\right\rangle \\
& +c r^{-C_{4}-\delta-3} \frac{f_{a}^{\prime}(r)}{f_{a}(r)}
\end{aligned}
$$




$$
\begin{aligned}
& \leq-c r^{-3 \delta-4}+c r^{-2 \delta-3} \frac{1}{f_{a}(r)}+c r^{-C_{4}-\delta-3} \frac{f_{a}^{\prime}(r)}{f_{a}(r)} \\
& \leq-c r^{-3 \delta-4}+c r^{-C_{4}-\delta-3} \frac{f_{a}^{\prime}(r)}{f_{a}(r)},
\end{aligned}
$$

where in the last step we have absorbed the term $c r^{-2 \delta-3} \frac{1}{f_{a}(r)}$ into the first by using the fact that $f_{a}(r) \geq c r^{\phi}$ and the choice of $\delta<\phi-1$. Putting together (5.14) and (5.15) we get

$$
-\frac{1}{2}\left\langle\nabla\left(\varrho^{-2}+|\nabla \psi|^{2}\right), \nabla \psi\right\rangle \leq-(1-\tilde{\varepsilon}) A R_{3}^{\delta} \delta r^{-\delta-1} \frac{\partial_{r} \varrho}{\varrho^{3}}-c r^{-3 \delta-4}+c r^{-C_{4}-\delta-3} \frac{f_{a}^{\prime}(r)}{f_{a}(r)},
$$

and combining this with (5.13) yields

$$
\begin{aligned}
\left(\varrho^{-2}\right. & \left.+|\nabla \psi|^{2}\right) \Delta_{-\log \varrho} \psi-\frac{1}{2}\left\langle\nabla\left(\varrho^{-2}+|\nabla \psi|^{2}\right), \nabla \psi\right\rangle \\
\leq & -\frac{A R_{3}^{\delta} \delta}{\varrho^{2}}\left(\frac{(1-\varepsilon)(n-1) r f_{a}^{\prime}(r)}{f_{a}(r)}+\frac{2(1-\tilde{\varepsilon}) r \partial_{r} \varrho}{\varrho}-\delta-1\right) r^{-\delta-2} \\
& -(1-\varepsilon)\left(A R_{3}^{\delta} \delta\right)^{3}\left(\frac{(1-\varepsilon)(n-1) r f_{a}^{\prime}(r)}{f_{a}(r)}+\frac{(1-\tilde{\varepsilon}) r \partial_{r} \varrho}{\varrho}-1-\delta+c\right) r^{-3 \delta-4},
\end{aligned}
$$

where we have absorbed the positive term $\mathrm{cr}^{-C_{4}-\delta-3} f_{a}^{\prime}(r) / f_{a}(r)$ by using the assumption $\delta<C_{4} / 2$. Finally, using the assumption (5.7) we can estimate the term involving the mean curvature as

$$
\begin{aligned}
- & \left(\varrho^{-2}+|\nabla \psi|^{2}\right)^{3 / 2} n H \\
\leq & (1+\varepsilon)^{3 / 2}\left(\varrho^{-2}+\left(A R_{3}^{\delta} \delta\right)^{2} r^{-2 \delta-2}\right)^{3 / 2} n|H| \\
\leq & \frac{c}{\varrho^{2}}\left(\frac{(n-1) r f_{a}^{\prime}(r)}{f_{a}(r)}+\frac{r \partial_{r} \varrho}{\varrho}-1\right) r^{-\delta_{1}-2} \\
& +c\left(\frac{(n-1) r f_{a}^{\prime}(r)}{f_{a}(r)}+\frac{r \partial_{r} \varrho}{\varrho}-1\right) r^{-2 \delta-\delta_{1}-4} .
\end{aligned}
$$

Combining (5.16) and (5.17) and noting that $\delta_{1}>\delta$ we obtain (5.10) and the claim follows.

Remark 5.6 In the case of the hyperbolic (ambient) space $\mathbb{H}^{n+1}=\mathbb{H}^{n} \times \cosh r$ Re have $\varrho=\varrho_{+}(r)=\cosh r$ and $f_{a}(r)=\sinh r$ on $\mathbb{H}^{n}$ for any reference point $o \in \mathbb{H}^{n}$. Hence (5.8) and (5.9) hold trivially. Moreover, we may choose $\phi>1$ as large as we wish by increasing $R_{3}$ and therefore (5.11) and (5.12) hold even with $\delta=\delta_{1}$. Finally,

$$
\left.-\left(\varrho^{-2}+|\nabla \psi|^{2}\right)^{3 / 2} n H \leq(1+\varepsilon)\left(A R_{3}^{\delta} \delta\right)^{3} r^{-3 \delta-3}\right) n|H|
$$

for $r$ large enough, and consequently we may assume $\delta=\delta_{1}$ in (5.7) thus reducing it to an asymptotically sharp assumption.

Similarly, if the sectional curvatures of $M$ have estimates

$$
-r(x)^{-2-\varepsilon} e^{2 k r(x)} \leq K\left(P_{x}\right) \leq-k^{2}
$$

for $r(x) \geq R_{0}$ as in Example 5.2 and if the warping function $\varrho$ satisfies (5.8), (5.9), and

$$
\varrho(x) \geq \operatorname{cr}(x)^{2}
$$

for $r(x) \geq R_{0}$, we may take $\delta=\delta_{1}$ in (5.7). 


\section{Solving the asymptotic Dirichlet problem}

In this section we solve the asymptotic Dirichlet problem (5.1) on a Cartan-Hadamard manifold $M$ with given boundary data $\varphi \in C\left(\partial_{\infty} M\right)$. If the ambient manifold $N=M \times_{\varrho} \mathbb{R}$ is a Cartan-Hadamard manifold, too, we will interpret the graph $S=\{(x, u(x)): x \in M\}$ of the solution $u$ as a Killing graph with prescribed mean curvature $H$ and continuous boundary values at infinity. We recall from $[2,7.7]$ that $N$ is a Cartan-Hadamard manifold if and only if the warping function $\varrho$ is convex. In that case we may consider $\partial_{\infty} M$ as a subset of $\partial_{\infty} N$ in the sense that a representative $\gamma$ of a boundary point $x_{0} \in \partial_{\infty} M$ is also a representative of a point $\tilde{x}_{0} \in \partial_{\infty} N$ since $M$ is a totally geodesic submanifold of $N$. Given $\varphi \in C\left(\partial_{\infty} M\right)$ we define its Killing graph on $\partial_{\infty} N$ as follows. For $x \in \partial_{\infty} M$, take the (totally geodesic) leaf

$$
M_{\varphi(x)}=\Psi(M, \varphi(x))=\{(y, \varphi(x)): y \in M\} \subset M \times \mathbb{R},
$$

where $\Psi$ is the flow generated by $X$. Let $\gamma^{x}$ be any geodesic on $M$ representing $x$. Then $\tilde{\gamma}^{x}: t \mapsto \Psi\left(\gamma^{x}(t), \varphi(x)\right)$ is a geodesic on $M_{\varphi(x)}$ and also on $N$ since $\Psi(\cdot, \varphi(x))$ is an isometry. Hence $\tilde{\gamma}^{x}$ defines a point in $\partial_{\infty} N$ which we, by abusing the notation, denote by $(x, \varphi(x))$. Using this notation, we call the set

$$
\Gamma=\left\{(x, \varphi(x)): x \in \partial_{\infty} M\right\} \subset \partial_{\infty} N
$$

the Killing graph of $\varphi$. Note that, in general, $\partial_{\infty} N$ has no canonical smooth structure.

Lemma 6.1 Let $u$ be the solution to (5.1) with boundary data $\varphi$ and let $S$ be the graph of $u$. If $\partial_{\infty} S=\bar{S} \backslash S$, where $\bar{S}$ is the closure of $S$ in the cone topology $\bar{N}$, we have $\partial_{\infty} S=\Gamma$.

Proof Suppose first that $x \in \partial_{\infty} S$ and let $\left(x_{i}, u\left(x_{i}\right)\right)$ be a sequence in $S$ converging to $x$ in the cone topology of $\bar{N}$. Since $\bar{M}$ is compact, there exist $x_{0} \in \partial_{\infty} M$ and a subsequence $\left(x_{i_{j}}, u\left(x_{i_{j}}\right)\right)$ such that $x_{i_{j}} \rightarrow x_{0} \in \partial_{\infty} M$ in the cone topology of $\bar{M}$. Hence $u\left(x_{i_{j}}\right) \rightarrow$ $\varphi\left(x_{0}\right)$, and consequently $\left(x_{i_{j}}, u\left(x_{i_{j}}\right)\right) \rightarrow\left(x_{0}, \varphi\left(x_{0}\right)\right)$ in the product topology of $\bar{M} \times \mathbb{R}$. On the other hand, $\Psi\left(x_{i_{j}}, \varphi\left(x_{0}\right)\right) \rightarrow\left(x_{0}, \varphi\left(x_{0}\right)\right)$ in the cone topology of $M_{\varphi\left(x_{0}\right)}$. We need to verify that $\Psi\left(x_{i_{j}}, u\left(x_{i_{j}}\right)\right) \rightarrow\left(x_{0}, \varphi\left(x_{0}\right)\right)$ in the cone topology of $\bar{N}$ which then implies that $x=\left(x_{0}, \varphi\left(x_{0}\right)\right) \in \Gamma$. Towards this end, let $V$ be an arbitrary cone neighborhood in $\bar{N}$ of $\left(x_{0}, \varphi\left(x_{0}\right)\right)$ and let $\sigma$ be a geodesic ray emanating from $\left(o, \varphi\left(x_{0}\right)\right)$ representing $\left(x_{0}, \varphi\left(x_{0}\right)\right)$. It is a geodesic ray both in $N$ and in $M_{\varphi\left(x_{0}\right)}$. Let $T\left(\dot{\sigma}_{0}, 2 \alpha, r\right) \subset V$ be a truncated cone in $\bar{N}$ and $T:=T^{M}\left(\dot{\sigma}_{0}, \alpha, 2 r\right)$ a truncated cone in $\bar{M}_{\varphi\left(x_{0}\right)}$. Then $\Psi\left(T,\left(\varphi\left(x_{0}\right)-\delta, \varphi\left(x_{0}\right)+\delta\right)\right) \subset V$ for sufficiently small $\delta>0$. It follows that $\Psi\left(x_{i_{j}}, u\left(x_{i_{j}}\right)\right) \in V$ for all $i_{j}$ large enough, and therefore $x=\left(x_{0}, \varphi\left(x_{0}\right)\right) \in \Gamma$.

Conversely, if $\left(x_{0}, \varphi\left(x_{0}\right)\right) \in \Gamma$, let $x_{i} \in M$ be a sequence such that $x_{i} \rightarrow x_{0}$ in the cone topology of $\bar{M}$. Then $\Psi\left(x_{i}, u\left(x_{i}\right)\right) \in S$ and $\left(x_{i}, u\left(x_{i}\right)\right) \rightarrow\left(x_{0}, \varphi\left(x_{0}\right)\right)$ in the product topology of $\bar{M} \times \mathbb{R}$. We need to show that $\Psi\left(x_{i}, u\left(x_{i}\right)\right) \rightarrow\left(x_{0}, \varphi\left(x_{0}\right)\right) \in \Gamma$ in the cone topology of $\bar{N}$. To prove this, fix $o=\Psi\left(x, \varphi\left(x_{0}\right)\right) \in M_{\varphi\left(x_{0}\right)}$ and let $\sigma$ be a geodesic ray in $N$ (and in $\left.M_{\varphi\left(x_{0}\right)}\right)$ representing $\left(x_{0}, \varphi\left(x_{0}\right)\right)$. Let $V=T\left(\dot{\sigma}_{0}, 2 \alpha, r\right)$ be an arbitrary truncated cone neighborhood in $\bar{N}$ of $\left(x_{0}, \varphi\left(x_{0}\right)\right)$. Furthermore, let $\delta>0$ be so small that $U:=\Psi\left(\tilde{V},\left(\varphi\left(x_{0}\right)-\delta, \varphi\left(x_{0}\right)+\delta\right)\right) \subset V$, where $\tilde{V}=T\left(\dot{\sigma}_{0}, \alpha, 2 r\right)$ is a truncated cone neighborhood in $M_{\varphi\left(x_{0}\right)}$ of $\left(x_{0}, \varphi\left(x_{0}\right)\right)$. Since $x_{i} \rightarrow x_{0}$ and $u\left(x_{i}\right) \rightarrow \varphi\left(x_{0}\right)$, we obtain $\Psi\left(x_{i}, u\left(x_{i}\right)\right) \in U$ for all sufficiently large $i$. Hence $\Psi\left(x_{i}, u\left(x_{i}\right)\right) \rightarrow\left(x_{0}, \varphi\left(x_{0}\right)\right) \in \Gamma$ in the cone topology of $\bar{N}$.

We formulate our global existence results in the following two theorems depending on the assumption on the prescribed mean curvature function $H$. 
Theorem 6.2 Let $M$ be a Cartan-Hadamard manifold satisfying the curvature assumptions (5.1) and (A1)-(A7) in Section 5. Furthermore, assume that the prescribed mean curvature function $H: M \rightarrow \mathbb{R}$ satisfies the assumptions (4.18) and (5.7) with a convex warping function $\varrho$ satisfying (4.13), (4.14), (5.8), and (5.9). Then there exists a unique solution $u: M \rightarrow \mathbb{R}$ to the Dirichlet problem

$$
\left\{\begin{array}{l}
\operatorname{div}_{-\log \varrho} \frac{\nabla u}{\sqrt{\varrho^{-2}+|\nabla u|^{2}}}=n H(x) \text { in } M \\
u \mid \partial_{\infty} M=\varphi
\end{array}\right.
$$

for any continuous function $\varphi: \partial_{\infty} M \rightarrow \mathbb{R}$.

Theorem 6.3 Let $M$ be a Cartan-Hadamard manifold satisfying the curvature assumptions (5.1) and (A1)-(A7) in Section 5. Furthermore, assume that the prescribed mean curvature function $H: M \rightarrow \mathbb{R}$ satisfies the assumptions (4.25) and (5.7) with a convex warping function $\varrho$ satisfying (4.19), (5.8), and (5.9). Then there exists a unique solution $u: M \rightarrow \mathbb{R}$ to the Dirichlet problem (5.1) for any continuous function $\varphi: \partial_{\infty} M \rightarrow \mathbb{R}$.

Proof The proofs of Theorems 6.2 and 6.3 are similar. The only difference is to use the global barrier $u_{+}$in Lemma 4.1 for 6.2 relative to $V$ in Lemma 4.3 for 6.3 .

Extend the boundary data function $\varphi \in C\left(\partial_{\infty} M\right)$ to a function $\varphi \in C(\bar{M})$ and let $B_{k}=$ $B(o, k), k \in \mathbb{N}$ be an exhaustion of $M$. Then by Corollary 2.2 there exist solutions $u_{k} \in$ $C^{2, \alpha}\left(B_{k}\right) \cap C\left(\bar{B}_{k}\right)$ to the Dirichlet problem

$$
\left\{\begin{array}{l}
\operatorname{div}_{-\log \varrho} \frac{\nabla u_{k}}{\sqrt{\varrho^{-2}+\left|\nabla u_{k}\right|^{2}}}=n H(x) \text { in } B_{k} \\
u_{k} \mid \partial B_{k}=\varphi
\end{array}\right.
$$

By Lemma 4.1, we see that the sequence $\left(u_{k}\right)$ is uniformly bounded. Applying the gradient estimates in compact domains and then the diagonal argument, we obtain a subsequence converging locally uniformly with respect to $C^{2}$-norm to a solution $u$. Next we show that $u$ extends continuously to the boundary $\partial_{\infty} M$ with $u \mid \partial_{\infty} M=\varphi$.

Let $x_{0} \in \partial_{\infty} M$ and $\varepsilon>0$ be fixed. By the continuity of the function $\varphi$ we find a constant $L \in(8 / \pi, \infty)$ so that

$$
\left|\varphi(y)-\varphi\left(x_{0}\right)\right|<\varepsilon / 2
$$

whenever $y \in C\left(v_{0}, 4 / L\right) \cap \partial_{\infty} M$, where $v_{0}=\dot{\gamma}_{0}^{o, x_{0}}$ is the initial direction of the geodesic ray representing $x_{0}$. Taking (4.16) into account, we can choose $R_{3}$ in Lemma 5.5 so big that $u_{+}(r) \leq\|\varphi\|_{\infty}+\varepsilon / 2$ when $r \geq R_{3}$.

We will show that

$$
w^{-}(x):=-\psi(x)+\varphi\left(x_{0}\right)-\varepsilon \leq u(x) \leq w^{+}(x):=\psi(x)+\varphi\left(x_{0}\right)+\varepsilon
$$

in the set $U:=3 \Omega \backslash \bar{B}\left(o, R_{3}\right)$. Here $\psi=A\left(R_{3}^{\delta} r^{-\delta}+h\right)$ is the supersolution from the Lemma 5.5 and $A=2\|\varphi\|_{\infty}$.

Again, by the continuity of the function $\varphi$ in $\bar{M}$, we can choose $k_{0}$ such that $\partial B_{k} \cap U \neq \varnothing$ and

$$
\left|\varphi(x)-\varphi\left(x_{0}\right)\right|<\varepsilon / 2
$$

for every $x \in \partial B_{k} \cap U$ when $k \geq k_{0}$. We denote $V_{k}=B_{k} \cap U$ for $k \geq k_{0}$ and note that

$$
\partial V_{k}=\left(B_{k} \cap \bar{U}\right) \cup\left(\partial U \cap \bar{B}_{k}\right) .
$$


We prove (5.2) by showing that

$$
w^{-} \leq u_{k} \leq w^{+}
$$

holds in $V_{k}$ for every $k \geq k_{0}$.

Let $k \geq k_{0}$ and $x \in \partial B_{k} \cap \bar{U}$. Since $u_{k}\left|\partial B_{k}=\varphi\right| \partial B_{k}$, (5.3) implies

$$
w^{-}(x) \leq \varphi\left(x_{0}\right)-\varepsilon / 2 \leq \varphi(x)=u_{k}(x) \leq \varphi\left(x_{0}\right)+\varepsilon / 2 \leq w^{+}(x) .
$$

By Lemma 5.3

$$
h \mid M \backslash\left(2 \Omega \cup B\left(o, R_{1}\right)\right)=1
$$

and since $R_{3}^{\delta} r^{-\delta}=1$ on $\partial B\left(o, R_{3}\right)$ we have

$$
\psi \geq A=2\|\varphi\|_{\infty}
$$

on $\partial U \cap B_{k}$. Since $u_{+}$from Lemma 4.1 is global supersolution with $u_{+} \geq\|\varphi\|_{\infty}$ on $\partial B_{k}$, the comparison principle gives $u_{k}\left|B_{k} \leq u_{+}\right| B_{k}$ and by the choice of $R_{3}$, we have

$$
u_{k} \leq\|\varphi\|_{\infty}+\varepsilon / 2
$$

in the set $B_{k} \backslash B\left(o, R_{3}\right)$.

Putting all together, it follows that

$$
w^{+}=\psi+\varphi\left(x_{0}\right)+\varepsilon \geq 2\|\varphi\|_{\infty}+\varphi\left(x_{0}\right)+\varepsilon \geq\|\varphi\|_{\infty}+\varepsilon \geq u_{k}
$$

on $\partial U \cap \bar{B}_{k}$. Similarly we have $u_{k} \geq w^{-}$on $\partial U \cap \bar{B}_{k}$ and therefore $w^{-} \leq u_{k} \leq w^{+}$on $\partial V_{k}$. By Lemma $5.5 \psi$ is a supersolution in $U$ and hence the comparison principle yields $u_{k} \leq w^{+}$in $U$. On the other hand, $-\psi$ is a subsolution in $U$, so $u_{k} \geq w^{-}$in $U$, and (5.4) follows. This is true for every $k \geq k_{0}$ so we have (5.2). Since $\lim _{x \rightarrow x_{0}} \psi(x)=0$, we have

$$
\limsup _{x \rightarrow x_{0}}\left|u(x)-\varphi\left(x_{0}\right)\right| \leq \varepsilon .
$$

The point $x_{0} \in \partial_{\infty} M$ and constant $\varepsilon>0$ were arbitrary so this shows that $u$ extends continuously to $C(\bar{M})$ and $u \mid \partial_{\infty} M=\varphi$. Finally, the uniqueness follows from the comparison principle.

\section{Non-existence result}

In the following, we state a non-existence result for the prescribed weighted mean curvature graph equation by adapting the approach of Pigola, Rigoli and Setti in [16]. We denote by $A(r)$ the area of the geodesic sphere $\partial B(o, r)$ centred at a fixed point $o \in M$.

Proposition 7.1 Let $p:[0, \infty) \rightarrow[0, \infty)$ be a continuous function such that for some $\bar{R}>0$ and for all $r \geq \bar{R}$ at least one of the following conditions is satisfied:

$$
\frac{\exp \left(D\left(\int_{0}^{r} \sqrt{p(s)} \mathrm{d} s\right)^{2}\right)}{\varrho_{0}(r)^{2} A(r)} \notin L^{1}(+\infty)
$$

for some constant $D>0$ and a smooth function $\varrho_{0}$, so that $\varrho(x) \leq \varrho_{0}(r(x))$, or

$$
\frac{\left(\int_{r}^{3 r / 2} \sqrt{p(s)} \mathrm{d} s\right)^{2}}{r \log \left(\varrho_{0}(2 r)^{2} \operatorname{vol}(B(o, 2 r))\right)} \geq h(r) \notin L^{1}(+\infty)
$$


with some continuous and monotonically non-increasing $h:[\bar{R}, \infty) \rightarrow(0, \infty)$. Let $u, v \in$ $C^{2}(M)$ satisfy

$$
\begin{aligned}
& \operatorname{div}_{-} \log \varrho \frac{\nabla u}{\sqrt{\varrho^{-2}+|\nabla u|^{2}}}-\operatorname{div}_{-} \log \varrho \frac{\nabla v}{\sqrt{\varrho^{-2}+|\nabla v|^{2}}}=q(x) \\
& \geq p(r(x)) \varrho_{0}(r(x)) \geq 0,
\end{aligned}
$$

and

$$
\sup _{M}(u-v)<+\infty
$$

Then, if $q \not \equiv 0$, there are no solutions to (5.3).

Proof The proof is very similar to that in [16], the only differences being our use of the divergence operator with respect to the weighted volume form $\varrho \mathrm{d} M$ and a suitable form of the Mikljukov-Hwang-Collin-Krust inequality which in our setting reads as follows

$$
\begin{aligned}
& \left\langle\frac{\nabla u}{\sqrt{\varrho^{-2}+|\nabla u|^{2}}}-\frac{\nabla v}{\sqrt{\varrho^{-2}+|\nabla v|^{2}}}, \nabla u-\nabla v\right\rangle \\
& \geq \frac{1}{2}\left(\sqrt{\varrho^{-2}+|\nabla u|^{2}}+\sqrt{\varrho^{-2}+|\nabla v|^{2}}\right)\left|\frac{\nabla u}{\sqrt{\varrho^{-2}+|\nabla u|^{2}}}-\frac{\nabla v}{\sqrt{\varrho^{-2}+|\nabla v|^{2}}}\right|^{2} \\
& \geq \varrho^{-1}\left|\frac{\nabla u}{\sqrt{\varrho^{-2}+|\nabla u|^{2}}}-\frac{\nabla v}{\sqrt{\varrho^{-2}+|\nabla v|^{2}}}\right|^{2} .
\end{aligned}
$$

Together these result in the extra factors of $\varrho_{0}$ in (5.1), (5.2), and on the right hand side of (5.3). Taking into account these differences the proof in [16] applies almost verbatim.

As direct corollaries of the previous theorem, we have

Corollary 7.2 Let $u$ be a bounded solution to

$$
\operatorname{div}_{-\log \varrho} \frac{\nabla u}{\sqrt{\varrho^{-2}+|\nabla u|^{2}}}=n H(x) \text { in } M,
$$

with $H \geq 0$.

(i) Suppose that $\varrho(x) \leq \varrho_{0}(r(x)) \leq r(x)^{\beta_{1}}, \beta_{1}>0$, and that $A(r) \leq r^{\beta_{2}}, \beta_{2}>0$, for large values of $r=r(x)$. Then

$$
\liminf _{r(x) \rightarrow \infty} H(x) \cdot \frac{r(x)^{2} \log r(x)}{\varrho_{0}(r(x))}=0 .
$$

(ii) Suppose that $\varrho(x) \leq \varrho_{0}(r(x)) \leq e^{\beta_{1} r(x)}, \beta_{1}>0$, and that $A(r) \leq e^{\beta_{2} r}, \beta_{2}>0$, for large values of $r=r(x)$. Then

$$
\liminf _{r(x) \rightarrow \infty} H(x) \cdot \frac{r(x) \log r(x)}{\varrho_{0}(r(x))}=0 .
$$

(iii) Suppose that $\varrho(x) \leq \varrho_{0}(r(x)) \leq e^{\beta_{1} r(x)^{2}}, \beta_{1}>0$, and that $A(r) \leq e^{\beta_{2} r^{2}}, \beta_{2}>0$, for large values of $r=r(x)$. Then

$$
\liminf _{r(x) \rightarrow \infty} H(x) \cdot \frac{\log r(x)}{\varrho_{0}(r(x))}=0 .
$$


Proof By choosing $p(s)=\left(s^{2} \log s\right)^{-1}$ in (i), we see that (5.1) holds, and therefore the claim follows. Similarly, choosing $p(s)=(s \log s)^{-1}$ in (ii) or $p(s)=(\log s)^{-1}$ in (iii), the condition (5.2) holds and the claim follows.

Acknowledgements Open access funding provided by University of Helsinki including Helsinki University Central Hospital.

Open Access This article is distributed under the terms of the Creative Commons Attribution 4.0 International License (http://creativecommons.org/licenses/by/4.0/), which permits unrestricted use, distribution, and reproduction in any medium, provided you give appropriate credit to the original author(s) and the source, provide a link to the Creative Commons license, and indicate if changes were made.

\section{References}

1. Anderson, M.T., Schoen, R.: Positive harmonic functions on complete manifolds of negative curvature. Ann. Math. (2) 121(3), 429-461 (1985)

2. Bishop, R.L., O'Neill, B.: Manifolds of negative curvature. Trans. Am. Math. Soc. 145, 1-49 (1969)

3. Bombieri, E., De Giorgi, E., Miranda, M.: Una maggiorazione a priori relativa alle ipersuperfici minimali non parametriche. Arch. Rational Mech. Anal. 32, 255-267 (1969)

4. Casteras, J.B., Heinonen, E., Holopainen, I.: Dirichlet problem for $f$-minimal graphs. J. Anal. Math., To appear

5. Casteras, J.B., Heinonen, E., Holopainen, I.: Existence and non-existence of minimal graphic and $p$ harmonic functions. Proc. R. Soc. Edinburgh Sect. A, To appear

6. Casteras, J.B., Holopainen, I., Ripoll, J.B.: Convexity at infinity in Cartan-Hadamard manifolds and applications to the asymptotic Dirichlet and Plateau problems. Math. Z. 290(1-2), 221-250 (2018)

7. Dajezer, M., de Lira, J.H.S.: Entire bounded constant mean curvature Killing graphs. J. Math. Pures Appl. (9) 103(1), 219-227 (2015)

8. Dajczer, M., de Lira, J.H.: Entire unbounded constant mean curvature Killing graphs. Bull. Braz. Math. Soc. (N.S.) 48(2), 187-198 (2017)

9. Dajczer, M., Ripoll, J.: An extension of a theorem of Serrin to graphs in warped products. J. Geom. Anal. 15(2), 193-205 (2005)

10. Dajczer, M., Hinojosa, P.A., de Lira, J.H.: Killing graphs with prescribed mean curvature. Calc. Var. Partial Differ. Equ. 33(2), 231-248 (2008)

11. Dajczer, M., de Lira, J.H., Ripoll, J.: An interior gradient estimate for the mean curvature equation of Killing graphs and applications. J. Anal. Math. 129,91-103 (2016)

12. Ding, Q., Jost, J., Xin, Y.: Minimal graphic functions on manifolds of nonnegative Ricci curvature. Commun. Pure Appl. Math. 69(2), 323-371 (2016)

13. Holopainen, I., Vähäkangas, A.: Asymptotic Dirichlet problem on negatively curved spaces. J. Anal. 15, 63-110 (2007)

14. Korevaar, N.: An easy proof of the interior gradient bound for solutions to the prescribed mean curvature equation. In Nonlinear functional analysis and its applications, Part 2 (Berkeley, Calif., 1983), volume 45 of Proc. Sympos. Pure Math., pages 81-89. Amer. Math. Soc., Providence, RI (1986)

15. Mastrolia, P., Monticelli, D.D., Punzo, F.: Elliptic and parabolic equations with Dirichlet conditions at infinity on Riemannian manifolds. Adv. Differ. Equ. 23(1-2), 89-108 (2018)

16. Pigola, S., Rigoli, M., Setti, A.G.: Some remarks on the prescribed mean curvature equation on complete manifolds. Pac. J. Math. 206(1), 195-217 (2002)

17. Ripoll, J., Telichevesky, M.: On the asymptotic Plateau problem for CMC hypersurfaces in hyperbolic space. Bull. Braz. Math. Soc. (N.S.) (2018)

18. Rosenberg, Harold, Schulze, Felix, Spruck, Joel: The half-space property and entire positive minimal graphs in $M \times \mathbb{R}$. J. Differ. Geom. 95(2), 321-336 (2013)

19. Spruck, J.: Interior gradient estimates and existence theorems for constant mean curvature graphs in $M^{n} \times \mathbf{R}$. Pure Appl. Math. Q., 3(3, Special Issue: In honor of Leon Simon. Part 2):785-800 (2007)

20. Wang, X.J.: Interior gradient estimates for mean curvature equations. Math. Z. 228(1), 73-81 (1998)

Publisher's Note Springer Nature remains neutral with regard to jurisdictional claims in published maps and institutional affiliations. 